\title{
Variability of solar/stellar activity and magnetic field and its influence on planetary atmosphere evolution
}

\author{
Helmut Lammer ${ }^{1}$, Manuel Güdel ${ }^{2}$, Yuri Kulikov ${ }^{3}$, Ignasi Ribas ${ }^{4}$, Teimuraz V. Zaqarashvili ${ }^{1,5}$, Maxim L. Khodachenko ${ }^{1}$, \\ Kristina G. Kislyakova ${ }^{6,7}$, Hannes Gröller ${ }^{1}$, Petra Odert ${ }^{7}$, Martin Leitzinger ${ }^{7}$, Bibiana Fichtinger ${ }^{7}$, Sandro Krauss ${ }^{1}$, \\ Walter Hausleitner ${ }^{1}$, Mats Holmström ${ }^{8}$, Jorge Sanz-Forcada ${ }^{9}$, Herbert I. M. Lichtenegger ${ }^{1}$, Arnold Hanslmeier ${ }^{7}$, \\ Valery I. Shematovich ${ }^{10}$, Dmitry Bisikalo ${ }^{10}$, Heike Rauer ${ }^{11,12}$, and Malcolm Fridlund ${ }^{13}$ \\ ${ }^{1}$ Space Research Institute, Austrian Academy of Sciences, Schmiedlstr. 6, A-8042 Graz, Austria \\ ${ }^{2}$ Institute for Astronomy, University of Vienna, Türkenschanzstr. 17, A-1180 Vienna, Austria \\ ${ }^{3}$ Polar Geophysical Institute, Russian Academy of Sciences, Khalturina Str. 15, 183010 Murmansk, Russia \\ ${ }^{4}$ Institut d'Estudis Espacials de Catalunya/CSIC, Campus UAB, Facultat de Ciències, Torre C5-parell-2a planta, 08193 Bellaterra, Spain \\ ${ }^{5}$ Abastumani Astrophysical Observatory at Ilia State University, Kazbegi ave. 2a, Tbilisi, Georgia \\ ${ }^{6}$ N. I. Lobachevsky State University, University of Nizhnij Novgorod, 23 Prospekt Gagarina, 603950 Nizhnij Novgorod, Russia \\ ${ }^{7}$ Institut für Physik/IGAM, Universität Graz Universitätsplatz 5, A-8010 Graz, Austria \\ ${ }^{8}$ Swedish Institute of Space Physics, Box 812, SE-98128 Kiruna, Sweden \\ ${ }^{9}$ Centro de Astrobiología/CSIC-INTA, LAEFF Campus, P.O. Box 78, E-28691 Villanueva de la Cañada, Madrid, Spain \\ ${ }^{10}$ Institute of Astronomy, Russian Academy of Sciences, 48 Pyatnitskaya Str., 119017 Moscow, Russia \\ ${ }^{11}$ Institut für Planetenforschung, Deutsches Zentrum für Luft- und Raumfahrt, Rutherfordstr. 2, 12489 Berlin, Germany \\ ${ }^{12}$ Zentrum für Astronomie und Astrophysik, TU Berlin, Hardenbergstr. 36, D-10623 Berlin, Germany \\ ${ }^{13}$ Research and Scientific Support Department, European Space Agency, Keplerlaan 1, 2200 AG, Noordwijk, The Netherlands
}

(Received January 31, 2011; Revised April 6, 2011; Accepted April 7, 2011; Online published March 8, 2012)

\begin{abstract}
It is shown that the evolution of planetary atmospheres can only be understood if one recognizes the fact that the radiation and particle environment of the Sun or a planet's host star were not always on the same level as at present. New insights and the latest observations and research regarding the evolution of the solar radiation, plasma environment and solar/stellar magnetic field derived from the observations of solar proxies with different ages will be given. We show that the extreme radiation and plasma environments of the young Sun/stars have important implications for the evolution of planetary atmospheres and may be responsible for the fact that planets with low gravity like early Mars most likely never build up a dense atmosphere during the first few 100 Myr after their origin. Finally we present an innovative new idea on how hydrogen clouds and energetic neutral atom (ENA) observations around transiting Earth-like exoplanets by space observatories such as the WSO-UV, can be used for validating the addressed atmospheric evolution studies. Such observations would enhance our understanding on the impact on the activity of the young Sun on the early atmospheres of Venus, Earth, Mars and other Solar System bodies as well as exoplanets.
\end{abstract}

Key words: Young Sun, solar activity, solar proxies, solar wind, atmosphere evolution, ENAs, WSO-UV, PLATO.

\section{Introduction}

The Sun has always played a major role in solar-planetary relations of planetary atmospheres. During the past decade the study of the history of our Sun and its influence on the evolution of Solar System planets has become an interdisciplinary effort between stellar astronomy, astrophysics of star and planet formation, astrochemistry, solar physics, geophysics, planetology, as well as climatological science. The great importance of understanding the historical evolution of our host star is obvious; the Sun's radiative energy, the plasma outflow, and various forms of transient phenomena such as shock waves, high-energy particle events during flares, and Coronal Mass Ejections (CMEs) are key factors in the formation and atmospheric evolution of the plan-

Copyright (C) The Society of Geomagnetism and Earth, Planetary and Space Sciences (SGEPSS); The Seismological Society of Japan; The Volcanological Society of Japan; The Geodetic Society of Japan; The Japanese Society for Planetary Sciences; TERRAPUB.

doi:10.5047/eps.2011.04.002 ets including Earth's biosphere (e.g., Lundin et al., 2007; Lammer et al., 2008, 2009).

The increase in solar luminosity over time and its climatological effect on Venus, Earth and Mars have been discussed by various authors in the past (e.g., Sagan and Mullen, 1972; Walker, 1975; Newman and Rood, 1977; Owen, 1979; Chassefière, 1996; Guinan and Ribas, 2002; Lammer et al., 2003; Ribas et al., 2005; Tian et al., 2005; Kulikov et al., 2006, 2007; Cnossen et al., 2007). The early Sun, with a luminosity of about $\sim 70 \%$ of that today, should have been too faint to prevent the early Earth and Mars from freezing. However, paleoclimate studies show that the young Earth always had liquid water and was therefore, typically warmer than during recent times and possibly heated by greenhouse gases such as $\mathrm{CO}_{2}, \mathrm{CH}_{4}$, and $\mathrm{H}_{2} \mathrm{O}$. Similar for Mars we know that our outer neighbor planet has traces of liquid $\mathrm{H}_{2} \mathrm{O}$-flows on its surface which indicates that there was once a time when Mars had a denser atmosphere and a 
warmer climate.

Besides the impact of the Sun on the planetary climate, solar radiation and particle fluxes play a tremendous role in the heating of the upper planetary atmospheres. The studies based on previous observation indicated that contrary to a weaker total luminosity the young Sun was a far stronger source of energetic particles and electromagnetic radiation (e.g., Newkirk Jr., 1980; Skumanich and Eddy, 1981; Zahnle and Walker, 1982). These early studies are now more and more supported by a large number of multiwavelength (X-ray, SXR, EUV, FUV, UV, optical) observations of solar proxies which are a solid evidence that the early Sun was a much more active star than it is at present (e.g., Ribas et al., 2005).

If the young Sun was more active than today, one has to study the consequences this might have had for the development of the planetary environments, including the formation and evolution of planetary atmospheres and initial $\mathrm{H}_{2} \mathrm{O}$ inventories. To understand the impacts of the radiation and particle environment of the young Sun or other stars on early planets and their potential habitability one has to focus on the following two main subjects:

- Young stars rotate more rapidly and have an internal magnetic dynamo that is more efficient than that of the present-day Sun. This results in stronger surface magnetic fields and/or higher surface magnetic filling factors which induce enhanced "activity" in all its variations, from larger surface spots to stronger, extended solar wind.

- The optical and infrared emissions of the young star largely control the planetary atmosphere and its related climate. The X-ray, SXR, EUV photons as well as the interaction with high-energy particles and the solar/stellar wind lead to atmospheric modifications which can be observed by satellites.

In this article we focus on the evolution of the solar/stellar radiation, plasma and magnetic environment during the Sun's (stars) life and its response on the upper atmospheres of terrestrial planets.

In Section 2 we discuss the latest research within the "Sun in time" project including uncertainties in stellar rotation periods, ages, radiation and plasma flow. Because the radiation and plasma history of our Sun can be separated into a period of a more moderate decrease from present to about 4 Gyr ago and a very early extreme period of about $500 \mathrm{Myr}$ after the young Sun arrived at the Zero-Age-Main-Sequence (ZAMS) we discuss its related radiation and plasma environment separately in Section 3 and Section 4. In Section 5 we discuss the latest theoretical findings on the impact of the extreme space weather of the young Sun on the evolution of the atmospheres of Venus, Earth and Mars and the consequences for terrestrial exoplanets orbiting stars with lower masses than the Sun. Finally we discuss in Section 6 a new innovative idea how one can test the atmospheric evolution theories addressed in Section 5 by transit observations of Earth-size exoplanets, which are exposed to higher SXR/EUV fluxes of dwarf stars.

\section{From "The Sun in Time" to "Stars in Time"}

In our understanding of the past magnetic evolution of the Sun, solar analogs or the so-called solar proxies, Sun-like stars with different ages and activity levels play an important role. For stars with masses $\leq 1.5 M_{\text {Sun }}$ and ages of about a few $100 \mathrm{Myr}$, angular momentum loss by a stellar wind brakes rotation in a way that correlates very well with the stellar age (e.g., Soderblom, 2010). The only independent variable, the stellar age, then determines the rotation period and, through the internal magnetic dynamo, magnetic activity at all levels of the stellar atmosphere, probably including characteristics of the stellar wind (Wood et al., 2002, 2005; Güdel, 2007).

The age determination of a star is one of the most complex issues in stellar astrophysics. Besides the age-rotation relation stellar ages can be inferred from memberships in moving groups or stellar clusters, isochrones, chromospheric activity (CaII HK indices), and astroseismology (e.g., Soderblom, 2010). Therefore, at best, estimates are coming from a variety of indicators that are used over different timescales. In this sense, it is very hard to associate error bars since systematic errors and not random errors are the dominating factors. Specifically for the rotation periods, their relative uncertainty is proportional to the period itself since longer periods mean less active stars and are thus harder to monitor because of the smaller modulation amplitude. For the "Sun in Time" stars shown in Table 1 rotation periods are known to better than $5 \%$ in all cases. For $\kappa^{1}$ Cet, for example, the rotation period which was determined by photometry with the MOST satellite has been determined to better than $1 \%$ (Walker et al., 2007). However, for an inactive star like $\beta$ Hyi, the determination is significantly poorer (around 10\%) (Walker et al., 2007).

The observation of and detailed research on a series of near-solar-mass stars back to ages close to the ZAMS-age is therefore sufficient to reconstruct the history of our Sun and the interaction of its magnetic activity with its overall environment. This is the main goal of the so-called "Sun in Time" project which was established more than a decade ago by Dorren and Guinan (1994a, b). Table 1 shows a sample of very well observed and studied solar proxies and their main physical parameters.

Key studies within the "Sun in Time" project refer to the following main points:

- Photospheric (optical) and UV evolution with time to characterize level and extent of surface magnetic activity studied with IUE (Messina and Guinan, 2002, 2003; Dorren and Guinan, 1994a).

- The FUV studied with FUSE to infer transition-region magnetic activity (Guinan et al., 2003).

- To obtain information on coronal activity the EUV was studied with EUVE (Ayres, 1997; Güdel et al., 1997; Audard et al., 1999; Ribas et al., 2005).

- To obtain full coverage in coronal temperatures and characterize flares, X-rays were studied by ROSAT and ASCA (Dorren et al., 1995; Güdel et al., 1995a, 1997; Güdel, 1997). For spectroscopic coverage X-rays have been investigated by XMM-Newton (Telleschi et al., 2005). 
Table 1. Physical parameters and age-rotation relation of well observed and studied solar proxies in comparison with the Sun (after Güdel, 2007). $L_{\mathrm{X}}$ refers to a range of $0.1-2.4 \mathrm{keV}$ as measured by the ROSAT satellite.

\begin{tabular}{|c|c|c|c|c|c|c|}
\hline Star name & Type & Mass $\left[M_{\text {Sun }}\right]$ & Radius $\left[R_{\text {Sun }}\right]$ & $\log \left(\frac{L_{X}}{L_{\text {bol }}}\right)$ & Rot. [days] & Age [Ga] \\
\hline HD 129333 [EK Dra] & $\mathrm{G} 1.5 \mathrm{~V}$ & 1.06 & 0.95 & -3.61 & 2.68 & 0.1 \\
\hline HD $72905\left[\pi^{1} \mathrm{UMa}\right]$ & G1.5 V & 1.03 & 0.95 & -4.47 & 4.9 & 0.3 \\
\hline HD 206860 [HN Peg] & G0 V & 1.06 & 0.99 & -4.52 & 4.86 & 0.3 \\
\hline HD $39587\left[\chi^{1}\right.$ Ori $]$ & G1 V & 1.01 & 0.96 & -4.65 & 5.24 & 0.3 \\
\hline HD 1835 [BE Cet] & G2 V & 0.99 & 1.02 & -4.46 & 7.65 & 0.6 \\
\hline HD $20630\left[\kappa^{1}\right.$ Cet $]$ & G5 V & 1.02 & 0.93 & -4.73 & 9.21 & 0.65 \\
\hline HD $114710[\beta \mathrm{Com}]$ & G0 V & 1.08 & 0.925 & -5.52 & 12 & 1.6 \\
\hline HD 190406 [15 Sge] & G5 V & 1.01 & 1.1 & -5.64 & 13.5 & 1.9 \\
\hline Sun & G2 V & 1 & 1 & -6.29 & 25.4 & 4.6 \\
\hline HD 146233 [18 Sco] & G2 V & 1.01 & 1.03 & - & 23 & 4.9 \\
\hline HD $128620[\alpha$ Cen A $]$ & G2 V & 1.1 & 1.22 & -6.67 & $\sim 30$ & $5-6$ \\
\hline HD 2151 [ $\beta$ Hyi] & G2 IV & 1.1 & 1.9 & -6.41 & $\sim 28$ & 6.7 \\
\hline HD 186408 [16 Cyg A] & G1.5 V & 1 & 1.16 & - & $\sim 35$ & 8.5 \\
\hline
\end{tabular}

- To measure the production of non-thermal particles in magnetic coronae the radio flux has been studied with the VLA (Güdel et al., 1995a, b, 1997; Gaidos et al., 2000; Güdel and Gaidos, 2001).

- Magnetic cycles of solar proxies (Messina and Guinan, 2002) are measured in the optical or in X-rays. Cycles were also studied for $\alpha$ Cen A a G2V star (Robrade et al., 2005; Ayres, 2009) and HD 81809 (G2V+G9V) (Favata et al., 2004, 2008) stars.

- Differential rotation of the solar proxies as seen in stars spot measurements were studied by Messina and Guinan (2003).

The much stronger magnetic activity of the young Sun resulted in increased flaring activity, CMEs and denser and more energetic solar wind and particle fluxes. For the study of atmospheric evolution and related thermal and nonthermal escape processes the evolution of the X-ray, SXR and EUV flux is of particular interest, because it can ionize atmospheric species and dissociate molecules, that would otherwise be relatively inert, thereby initiating photochemistry that can alter an atmosphere's bulk composition as well as creating a class of radiation-dependent trace chemicals. Furthermore as a result of these photochemical processes, this wavelength range controls the temperature structure and expansion of the upper atmosphere and should have played an important role for large-scale atmospheric escape and isotopic fractionation on early Venus, Earth, and Mars.

Because lower-mass stars are very common and hence may host rocky planets within their habitable zones, progress in this direction has started by expanding the "Sun in Time" project to time sequences of the high-energy emissions, CME activity, stellar winds, and flare activity to lower-mass $\mathrm{K}$ and M-type stars. These stars are far more numerous than the solar type stars, have long mainsequence lifetimes, and have become prime targets for terrestrial planet searches in recent years. Because of the low luminosities, their habitable zones (e.g., Kasting et al., 1993; Lammer et al., 2009) can be quite close to the host stars. Because these dwarf stars have deeper outer convective zones than Sun-like stars and thus possess very efficient magnetic dynamos, the expected enhanced X-ray,
SXR, EUV radiation environment combined with extreme space weather effects should play a major role in the development of the atmospheres and habitability on such planets (Scalo et al., 2007; Lammer et al., 2009).

\section{The Moderate Activity Period: Solar Radia- tion, Plasma and Magnetic Environment From the Present to 4 Gyr Ago}

Ultraviolet emission from the Sun and late-type stars originates in magnetic chromospheric and transition-zone regions which have been heated to temperatures of $\sim 10^{4}$ $10^{5} \mathrm{~K}$. Based on spectral measurements obtained by IUE, Dorren and Guinan (1994a) have studied the evolution of ultraviolet (UV) line fluxes in detail and Güdel et al. (1997) and Ribas et al. (2005) extended this research to X-rays, SXR and EUV. The relevant wavelengths for thermospheric heating are the "ionizing" ones $\leq 1000 \AA$ (e.g., Hunten, 1993), which contain only a small fraction of the present solar spectral power. Planetary atmospheres are opaque to EUV radiation (100-920 $\AA$ ), as in the case of Earth, due to photo-absorption by $\mathrm{N}_{2}, \mathrm{O}_{2}$ and $\mathrm{O}$, so that the optical depth of unity at $100 \AA$ occurs at an altitude of about $130 \mathrm{~km}$.

Molecular constituents such as molecular hydrogen, carbon dioxide, molecular oxygen and $\mathrm{N}_{2}$ (Bakalian, 2006) can also be dissociated by relatively long wavelengths in the "Schumann-Runge" range of 1750-1300 ̊, according to

$$
\begin{aligned}
& \mathrm{O}_{2}+h v(\lambda<1750 \AA) \rightarrow \mathrm{O}\left({ }^{3} \mathrm{P}\right)+\mathrm{O}\left({ }^{1} \mathrm{D}\right), \\
& \mathrm{CO}_{2}+h v(\lambda<1670 \AA) \rightarrow \mathrm{CO}+\mathrm{O}\left({ }^{1} \mathrm{D}\right) .
\end{aligned}
$$

The solar SXR and EUV radiation with an intensity of $\geq 2 \mathrm{erg} \mathrm{cm}^{-2} \mathrm{sec}^{-1}$ represents the predominant heat source for Earth's upper atmosphere. Dissociation can also take place below the true dissociation limit due to excitation of molecules into a state which dissociates.

Dissociation of molecular ions and ion-neutral reactions produce so-called "hot" atoms which can reach the upper thermosphere and exobase where they form planetary coronae which can be ionized and picked up by the solar wind plasma flow (e.g. Nagy et al., 1981; Lammer and Bauer, 1991; Kim et al., 1998; Hodges, 2000; Krestyanikova and 
Table 2. Solar photon fluxes for moderate solar activity at $1 \mathrm{AU}$ (Bauer and Lammer, 2004).

\begin{tabular}{l|cc}
\hline \hline$\lambda[\AA]$ & $\Phi_{\infty}\left[10^{9} \mathrm{ph} \mathrm{cm}^{-2} \mathrm{sec}^{-1}\right]$ & $I_{\infty}\left[\mathrm{erg} \mathrm{cm}^{-2} \mathrm{sec}^{-1}\right]$ \\
\hline 1215.7 (Ly $\alpha)$ & 300 & 5 \\
$1027-911$ & 11.61 & 0.23 \\
1025.7 (Ly $\beta)$ & 3.5 & 0.067 \\
977 (C III) & 4.4 & 0.090 \\
$911-800$ & 8.3 & 0.20 \\
$800-630$ & 2.4 & 0.064 \\
$630-460$ & 4.7 & 0.17 \\
584.3 (He I) & 0.9 & 0.03 \\
$460-370$ & 0.63 & 0.03 \\
$370-270$ & 10.3 & 0.65 \\
303.8 (He II) & 5.4 & 0.35 \\
$270-205$ & 4.5 & 0.36 \\
$205-153$ & 4.6 & 0.49 \\
$153-100$ & 0.4 & 0.06 \\
$120-80$ & 0.3 & 0.066 \\
$80-40$ & 0.33 & 0.108
\end{tabular}

Table 3. Solar minimum and maximum values compared to the intensity flux of medium solar activity cycle for X-ray, SXR, EUV, FUV and Lyman-alpha wavelength ranges.

\begin{tabular}{|c|c|c|}
\hline Wavelength & High solar activity & Low solar activity \\
\hline X-ray $[1-20 \AA]$ & $\sim 2.6$ & $\sim 0.4$ \\
\hline SXR [20-100 ̊̊] & $\sim 1.8$ & $\sim 0.7$ \\
\hline EUV [100-912 Å] & $\sim 1.5$ & $\sim 0.8$ \\
\hline FUV [912-1180 ̊] & $\sim 1.3$ & $\sim 0.8$ \\
\hline Lyman- $\alpha$ [1200-1300 Å] & $\sim 1.3$ & $\sim 0.9$ \\
\hline
\end{tabular}

Shematovich, 2006; Lammer et al., 2006; Lichtenegger et al., 2009; Gröller et al., 2010; Valeille et al., 2010).

For $\mathrm{H}_{2}$, radiation $\lambda<850 \AA$ leads to either dissociation or ionization. Atomic hydrogen possesses a strong continuum absorption cross section below $912 \AA$ and strong Lyman-series lines, principally the intense Lyman- $\alpha$ line at $121.6 \mathrm{~nm}$. On Earth, rocket and satellite observations have led to a detailed identification of the solar emission line spectrum; relatively accurate values for the intensities of these radiations have become available by now (e.g., Hall et al., 1969; Tobiska, 1993; Tobiska and Eparvier, 1998). Solar photon fluxes at Earth for moderate solar activity are summarized in Table 2 . The solar photon flux $\phi_{\infty}=\lambda I_{\infty} /(h c)$ with $h$ the Plank constant and $c$ the speed of light is used for the ionization process, while the SXR and EUV energy flux $I_{\infty}=(h \nu) \phi_{\infty}$ is relevant for heating of the upper atmosphere.

\subsection{Short- and longtime variation of the short wave- length radiation}

The solar photon fluxes vary over the 11 year solar cycle as well as over the $\approx 27$ day solar rotation period owing to magnetic field inhomogeneities. Additionally to the photon flux variation, also the solar intensity fluxes differ in phase with the solar cycle. The Solar Irradiance Platform (SIP) ${ }^{1}$, which produces full solar spectra for any observation date, can be used to obtain intensity fluxes in the SXR, EUV and FUV wavelength ranges. The results, representing quiet and

\footnotetext{
${ }^{1}$ http://www.spacewx.com/solar2000.html
}

active conditions on the Sun normalized to the average flux value, are listed in Table 3 .

To understand the evolution of the solar radiation beyond activity levels which can be generated during high solar activity one has to analyze solar proxies which are observed in the "Sun in Time" project. As can be seen in Table 1, the sample of solar proxies contains stars that cover most of the Sun's main sequence lifetime from $\sim 100 \mathrm{Myr}$ to 8.5 Gyr. The observations, obtained with the space observatories shown in Table 4, cover a wide range between $1 \AA$ and $8300 \AA$.

One should note that the actual spectra covered the wavelength range $80-400 \AA$ in the best cases but some stellar spectra were still noisy well below $400 \AA$, due to the high opacity of the interstellar medium at these wavelengths. Therefore, the flux in the EUV range (100-912 A) given in Tables 5 and 7 was partly interpolated. The approach is explained in detail in Ribas et al. (2005). A promising alternative to the interpolation was applied by Sanz-Forcada $e t$ al. (2011) to a sample of stars with exoplanets: the X-rays and UV spectral information is used to calculate a model of the corona and transition region, the stellar layers where the XUV emission originates; this model is then used to synthetize the spectral energy distribution in the whole XUV range. The application of this approach to the two solar ancestors EK Dra and $\kappa$ Cet shows less than 0.15 dex of disagreement with the EUV flux calculated by Ribas et al. (2005).

It should be mentioned that the present solar activity 
Table 4. Space observatories and instruments used for the relevant observations within the "Sun in Time" project (Güdel, 2007).

\begin{tabular}{l|cc}
\hline \hline Space observatory & $\lambda[\AA]$ & $E[\mathrm{keV}]$ \\
\hline XMM-Newton [CCDs] & $0.83-83$ & $0.15-15$ \\
XMM-Newton [gratings] & $6-38$ & $0.33-2.1$ \\
ASCA [CCDs] & $1.2-31$ & $0.4-10$ \\
ROSAT & $5.2-124$ & $0.1-2.4$ \\
EUVE [gratings] & $80-760$ & $0.016-0.15$ \\
FUSE [gratings] & $920-1180$ & - \\
HST [gratings] & $1150-1730$ & - \\
IUE [gratings] & $1150-1950$ & - \\
UBVRI [photometry] & $3500-8300$ & -
\end{tabular}

Table 5. Solar radiation flux enhancement as function of wavelength normalized to the present solar flux value from present to 3.9 Gyr ago (Ribas $e t$ al., 2005; Güdel, 2007).

\begin{tabular}{l|cccccc}
\hline \hline $\begin{array}{l}\text { Solar age } \\
{[\mathrm{Gyr}]}\end{array}$ & $\begin{array}{c}t \text { b.p. } \\
{[\mathrm{Gyr}]}\end{array}$ & $\begin{array}{c}\text { X-ray } \\
{[1-20 \AA]}\end{array}$ & $\begin{array}{c}\text { SXR } \\
{[20-100 \AA]}\end{array}$ & $\begin{array}{c}\text { EUV } \\
{[100-920 \AA]}\end{array}$ & $\begin{array}{c}\text { FUV } \\
{[920-1180 \AA]}\end{array}$ & $\begin{array}{c}\text { Lyman- } \alpha \\
{[1200-1300 \AA]}\end{array}$ \\
\hline 4.6 & 0 & 1 & 1 & 1 & 1 & 1 \\
3.2 & 1.4 & 2 & 1.6 & 1.5 & 1.4 & 1.3 \\
2.6 & 2 & 3 & 2 & 1.9 & 1.6 & 1.5 \\
1.9 & 2.7 & 6 & 3 & 2.7 & 2.1 & 1.9 \\
1.1 & 3.5 & 16 & 6 & 5.1 & 3.4 & 2.8 \\
0.7 & 3.9 & 37 & 11 & 8.6 & 5.9 & - \\
& & & & & &
\end{tabular}

also shows intermediate periodicities in the range of several months (Rieger et al., 1984) and $\sim 2$ years (Vecchio et al., 2010). These periodicities are explained in terms of a Rossby wave instability in the solar interior (Zaqarashvili et al., 2010a, b). Similar periodicities have been observed in other solar like stars (Massi et al., 1998). Recently, Lanza et al. (2009) observed short term cyclic oscillations of the total spotted area on the photosphere of CoRoT-Exo-2a, which has been suggested to be caused by Rossby waves. The Rossby wave activity was probably enhanced in the early stage of solar evolution due to rapid rotation, therefore this aspect requires future detailed study.

The total high energy output of a stellar corona depends on the available magnetic energy and is therefore a consequence of the star's dynamo operation. Younger and more rapidly rotating stars are more X-ray luminous. A similar behavior can be observed for UV and FUV radiation; the $\mathrm{X}$-ray output decreases as the star ages and its rotation period increases. Table 5 lists the short wavelength radiation fluxes of the Sun as a function of stellar age between 0.7 and 4.6 Gyr. The fluxes are divided in six wavelength intervals are normalized to the present solar values. The radiation flux-age correlation can be expressed by the following power laws (Ribas et al., 2005)

$$
\begin{gathered}
F_{\mathrm{X}-\text { ray }}=2.4 t^{-1.92}, \\
F_{\mathrm{SXR}}=4.45 t^{-1.27}, \\
F_{\mathrm{EUV}}=13.5 t^{-1.2}+4.56 t^{-1.0}, \\
F_{\mathrm{FUV}}=2.53 t^{-0.85}, \\
F_{\mathrm{Ly}-\alpha}=19.2 t^{-0.72},
\end{gathered}
$$

where $F$ is the flux at $1 \mathrm{AU}$ in $\mathrm{erg} \mathrm{cm}^{-2} \mathrm{~s}^{-1}$ and $t$ the stellar age in Gyr. As Ribas et al. (2005) give no power laws for the evolution of fluxes longward of $1200 \AA$, we used their equation (2) and the observational data given in their table 6 to estimate the flux evolution between 1200 1700 A. Ribas et al. (2005) give observed emission line fluxes of six solar analogs (including the Sun), eight lines have wavelengths $>1200 \AA$. The major flux contributor in the $1200-1300 \AA$ range is the Lyman- $\alpha$ line (1216 $\AA$ ), but unfortunately it has only been measured for three stars. As the flux of the Si III line (1206 $\AA$ ) is negligible compared to Lyman- $\alpha$, we use the Lyman- $\alpha$ power law as a proxy for the whole 1200-1300 $\AA$ range. The range between 1300-1700 $\AA$, which approximately covers the important "Schumann-Runge" range, contains six strong emission lines. One can see from Table 3 that the Sun during its maximum solar cycle can be used as a proxy for the Sun when it had a similar flux in average quiet activity conditions $\sim 1.5-2$ Gyr ago.

\subsection{The solar magnetic field}

Rotation and convection lead to the solar dynamo activity, which results in complex magnetic fields in the solar interior and atmosphere. Rotation rate and convective mixing length have been continuously changing during the solar evolution, therefore the magnetic field structure in the solar atmosphere/corona was most likely different in the early stage of solar evolution from what is observed today. Young solar analogs with fast rotation $\leq 5$ days show polar spots in addition to the present day low latitude Sunspots (Strassmeier, 2009). It has been suggested that a dominance of the Coriolis force over the buoyancy force in the dynamics of rising magnetic flux tubes tends to the polar appearance of magnetic flux in fast rotators (Schüssler and Solanki, 1992; Schüssler et al., 1996). On the other hand, the meridional advection also may lead to the transport of photospheric magnetic flux towards the polar regions (Schrijver and Title, 2001; Mackay et al., 2004). 
Table 6. Stars with mass loss rates determined from Ly $\alpha$ absorption. Spectral types and mass loss rates taken from Wood et al. (2005). Ages determined via gyrochronology $\left(t_{\text {gyro }}\right)$, chromospheric activity $\left(t_{\text {chromo }}\right)$ and isochrones $\left(t_{\text {iso }}\right)$ were taken from Barnes $(2007)$. Other ages $\left(t_{\text {other }}\right)$ are taken from the references in the last column ([1] Eggenberger et al. (2004); [2] Soderblom and Mayor (1993); [3] King et al. (2010); [4] Eggen (1996); [5] DeWarf et al. (2010); [6] Eggenberger et al. (2008); [7] Montes et al. (2001)).

\begin{tabular}{|c|c|c|c|c|c|c|c|}
\hline Star & $\begin{array}{c}\text { Spectral } \\
\text { type }\end{array}$ & $\begin{array}{c}\dot{M} \\
\left(\dot{M}_{\odot}\right)\end{array}$ & $\begin{array}{c}t_{\text {gyro }} \\
(\mathrm{Gyr})\end{array}$ & $\begin{array}{c}t_{\text {chromo }} \\
(\mathrm{Gyr})\end{array}$ & $\begin{array}{c}t_{\text {iso }} \\
(\mathrm{Gyr})\end{array}$ & $\begin{array}{l}t_{\text {other }} \\
(\mathrm{Gyr})\end{array}$ & Method \& Ref. \\
\hline$\alpha$ Cen & $\mathrm{G} 2 \mathrm{~V}+\mathrm{K} 0 \mathrm{~V}$ & 2 & $4.4 \pm 0.5$ & 5.62 & 7.84 & $6.5 \pm 0.3$ & Astroseismology [1] \\
\hline $61 \mathrm{Vir}$ & G5V & 0.3 & $4.2 \pm 0.7$ & 5.6 & 8.96 & - & - \\
\hline$\xi$ Boo & $\mathrm{G} 8 \mathrm{~V}+\mathrm{K} 4 \mathrm{~V}$ & 5 & $0.23 \pm 0.02$ & 0.23 & $<0.76$ & 0.3 & UMa moving group [2] \\
\hline$\epsilon$ Ind & $\mathrm{K} 5 \mathrm{~V}$ & 0.5 & $1.0 \pm 0.1$ & 一 & - & $4 \pm 0.3$ & Age of BD companion [3] \\
\hline 61 Cyg A & $\mathrm{K} 5 \mathrm{~V}$ & 0.5 & $2.0 \pm 0.2$ & 2.36 & $<0.44$ & $6 \pm 2$ & 61 Cyg moving group [4] \\
\hline 36 Oph & $\mathrm{K} 1 \mathrm{~V}+\mathrm{K} 1 \mathrm{~V}$ & 15 & $0.59 \pm 0.07$ & 1.14 & - & 2 & Activity-rotation [5] \\
\hline $70 \mathrm{Oph}$ & $\mathrm{K} 0 \mathrm{~V}+\mathrm{K} 5 \mathrm{~V}$ & 100 & $1.3 \pm 0.2$ & 1.09 & - & $6.2 \pm 1$ & Astroseismology [6] \\
\hline$\epsilon$ Eri & K1V & 30 & $0.44 \pm 0.05$ & 0.66 & $<0.6$ & 0.65 & Hyades supercluster [5] \\
\hline Proxima Cen & M5.5V & $<0.2$ & $4.4 \pm 0.5$ & 5.62 & 7.84 & $6.5 \pm 0.3$ & Ages adopted from $\alpha$ Cen \\
\hline EV Lac & $\mathrm{M} 3.5 \mathrm{~V}$ & 1 & - & - & - & 0.3 & UMa moving group [7] \\
\hline
\end{tabular}

High-latitude appearance of Sun-spots could change the solar wind structure (possible interchange of fast and slow solar wind locations) and may also have affected the initiation latitudes of solar flares/CMEs. This could completely change the solar activity patterns, butterfly diagram and influence of solar wind/CMEs on the Solar System. However, this would have happened in the very early stages of the solar evolution, when the Sun just arrived at the ZAMS. The solar rotation period was $\sim 10$ days 4 Gyr ago (see Table 1 ), which is faster than the nowadays Sun, but still slower than at the time when the young Sun arrived at the ZAMS. This rotation rate does not affect too much on appearance of sunspot and therefore the magnetic structure of solar atmosphere/corona probably has not changed significantly during the past $4 \mathrm{Gyr}$.

\subsection{The time evolution of the solar wind}

As a consequence of solar/stellar activity, plasma winds are ejected from the star but their detection in solar proxies is very difficult. Although the wind formation and acceleration mechanisms are still not completely understood at the Sun, there is much evidence that magnetic fields play an important role for acceleration of the wind, for its heating, or for guiding the wind at least out to the Alfvén radius at which the stellar wind speed reaches the value of the Alfvén speed. Beyond the Alfvén radius the stellar wind is superAlfvénic. One of the best indirect confirmation of the presence of magnetized winds in young stars is their spin-down to slower rotation (as shown in Table 1) after their arrival at the ZAMS because the outward flowing plasma carries away angular momentum from the star.

Potential detection methods of stellar winds include the measurement of thermal radio emission from the plasma flow (Lim and White, 1996; Gaidos et al., 2000), and signatures of charge exchange in X-ray spectra (Wargelin and Drake, 2001). The most successful approach so far is an indirect method which makes use of the Lyman- $\alpha$ absorption in astrospheres due to the interaction between stellar winds and the interstellar medium (ISM) (e.g., Wood, 2004). When stellar winds collide with the ISM, at further distance from the star, a termination shock where the wind is shocked to subsonic speeds is formed. The heliosphere is permeated by interstellar $\mathrm{H}$ I with $T \sim(2-4) \times 10^{4} \mathrm{~K}$
(Wood, 2004; Wood et al., 2005). Much of this gas is piled up between the heliopause and the bow shock producing a hydrogen wall that can be detected by UV observations as an absorption signature in the Lyman- $\alpha$ line. The excess absorption from the Sun's own hydrogen wall is, due to the deceleration of the ISM relative to the star, redshifted, while that of astrospheres are blueshifted. The measurable absorption depths are then compared with results from hydrodynamic model calculations (Wood et al., 2005). We note that the the amount of astrospheric absorption scales with the wind ram pressure, and the stellar wind velocity has to be assumed to derive the mass loss rate (Wood and Linsky, 1998).

A systematic study of all derived mass-loss rates of main sequence stars which were observed with this method indicates that $d M_{\mathrm{L}} / d t$ per unit stellar surface correlates with stellar activity and hence the stellar X-ray surface flux $F_{\mathrm{X}}$ (Wood et al., 2005)

$$
\frac{d M_{\mathrm{L}}}{d t} \propto F_{\mathrm{X}}^{1.34 \pm 0.18}
$$

If we extrapolate the above power law up to the observed $\mathrm{X}$-ray saturation limit $F_{\mathrm{X}_{\text {sat }}}$ of Sun-like stars of about $2 \times$ $10^{7} \mathrm{erg} \mathrm{cm}^{-2} \mathrm{~s}^{-1}$, we would suggest that the mass loss of plasma from the youngest stars in Table 1 is about 1000 times larger compared to the present day Sun. However, recent observations indicate that this power law relation breaks down for the most active stars for which $F_{\mathrm{X}} \geq$ $8 \times 10^{5} \mathrm{erg} \mathrm{cm}^{-2} \mathrm{~s}^{-1}$ (Wood et al., 2005).

Table 6 shows the stars observed by Wood et al. (2005) and their mass loss rates in units of the solar mass loss rate. Note that only one star from the "Sun in Time" sample in Table 1 has a measured mass loss rate ( $\alpha$ Cen). If two spectral types are given in the second column, this indicates that two stars share the same astrosphere and the mass loss rate is a combined value for two stars. Age estimates for the stars as determined by different methods are also given. These include ages from gyrochronology (i.e. rotation-age relations), chromospheric activity, isochrones, and other methods, which include astroseismology, membership in moving groups, or known ages of binary companions. The sometimes discrepant results illustrate the difficulties in a 


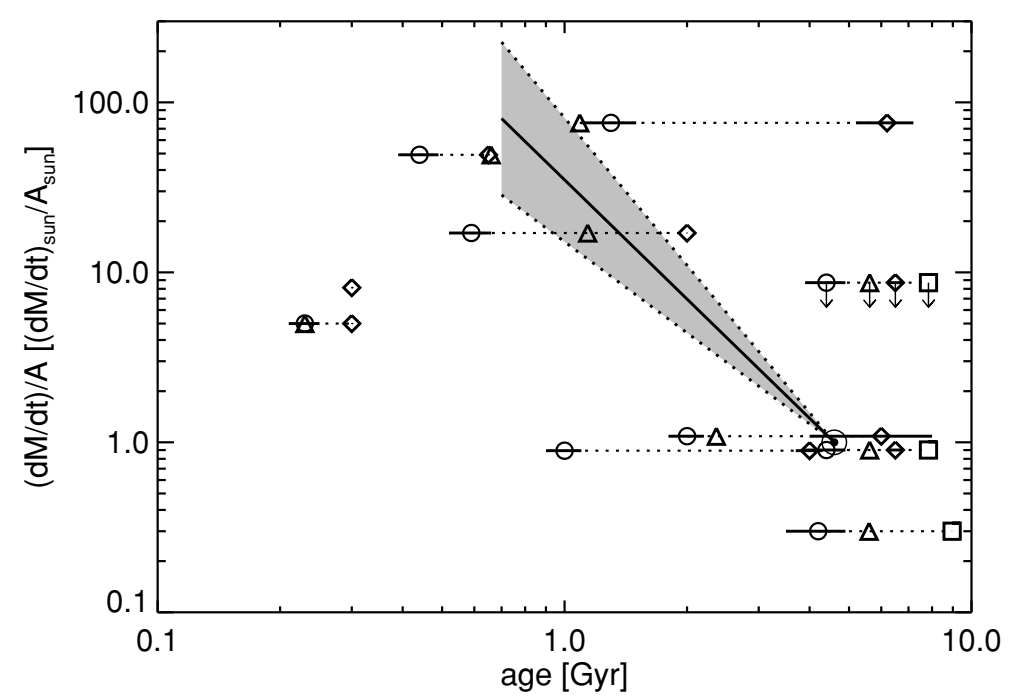

Fig. 1. Mass loss rates normalized to stellar surface area as a function of age for stars in Table 6. The adopted mass loss rate of the Sun is $2 \times 10^{-14} M_{\odot} \mathrm{yr}^{-1}$. The shaded area denotes the evolution $\dot{M} \propto t^{-2.33 \pm 0.55}$ derived from Eq. (3) and $F_{\mathrm{X}} \propto t^{-1.74 \pm 0.34}$ (Ayres, 1997) by Wood $e t$ al. (2005). The different symbols (circle, triangle, square, diamond) represent different age determination methods $\left(t_{\mathrm{gyro}}, t_{\mathrm{chromo}}, t_{\mathrm{iso}}, t_{\mathrm{other}}\right.$; cf. Table 6$)$.

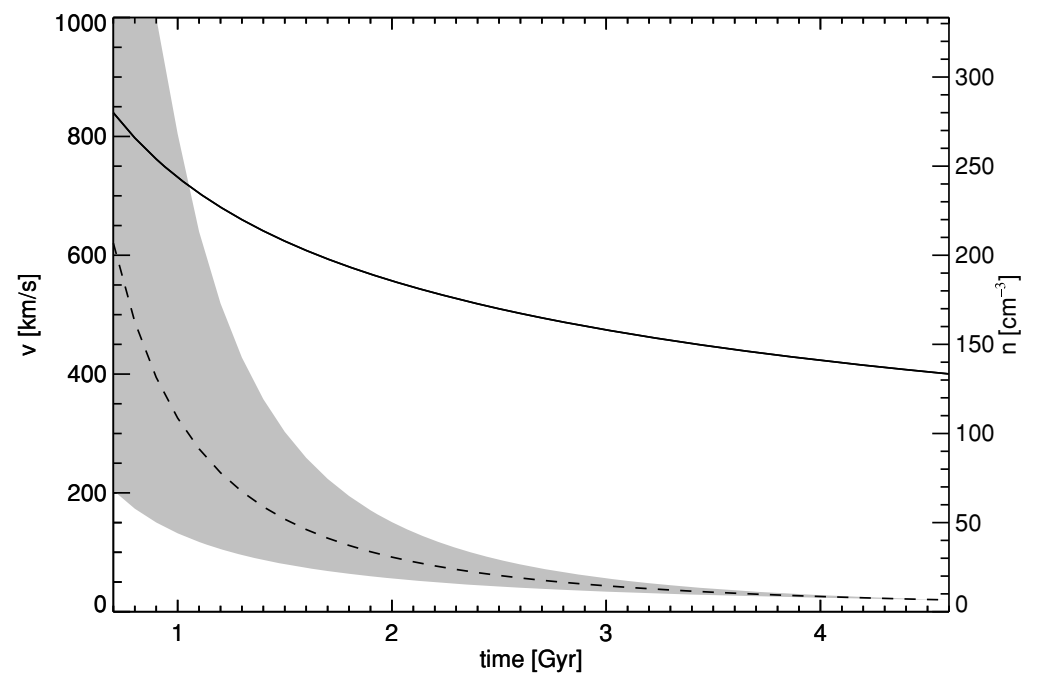

Fig. 2. Average stellar wind density (dashed line) and velocity (solid line) expected at 1 AU from present time (4.6 Gyr) to 0.7 Gyr, according to mass loss observations of Sun-like stars (Wood et al., 2005) and the power laws of Newkirk (1980). The shaded area represents to uncertainties of the solar wind density in time.

reliable determination of stellar ages. Figure 1 shows the stellar mass loss rates as a function of age. The mass loss rates are normalized to stellar surface area to simplify comparison between stars with different spectral types, as well as two stars sharing the same astrosphere. Ages determined by different methods for the same star are indicated by different symbols and connected by dotted lines. The shaded area denotes the mass loss evolution derived by Wood et $a l$. (2005), who used their power law fit to the X-ray surface flux (cf. Eq. (3)) and the evolution of the X-ray flux $\left(F_{\mathrm{X}} \propto t^{-1.74 \pm 0.34}\right)$ of Sun-like stars (Ayres, 1997) to construct the scaling $\dot{M} \propto t^{-2.33 \pm 0.55}$. As one can see from Fig. 1 stars at the lower age (i.e., high activity) limit indicate a mass loss which is about 100 times the present solar value, while the mass loss drops toward higher activity levels to about 10 times the solar value. We will discuss the possible reason for this breakdown between X-ray activity and wind-mass loss relation in the next section in more detail.

For studying the effect on planetary environments against solar/stellar wind induced non-thermal escape, the solar/stellar wind density $n_{\mathrm{sw}}$ and velocity $v_{\mathrm{sw}}$ in the vicinity of a planet are key parameters. These parameters for a Sun-like star can be estimated as a function of the age from the observations discussed above, together with an empirical model for an age-dependence of the solar/stellar wind velocity (Newkirk Jr., 1980; Grießmeier et al., 2004, 2007; Lichtenegger et al., 2010)

$$
v_{\mathrm{sw}}=v_{*}\left(1+\frac{t}{\tau}\right)^{-0.4}
$$


the minimum estimated solar/stellar wind density

$$
n_{\min _{\mathrm{sw}}}(t)=n_{\min *}\left(1+\frac{t}{\tau}\right)^{-1.26},
$$

an average solar/stellar wind density

$$
n_{\mathrm{ave}_{\mathrm{sw}}}(t)=n_{\mathrm{ave} *}\left(1+\frac{t}{\tau}\right)^{-1.86}
$$

and maximum solar/stellar wind density

$$
n_{\max _{\mathrm{sw}}}(t)=n_{\max *}\left(1+\frac{t}{\tau}\right)^{-2.46} .
$$

The proportionality constants $\left(v_{*}, n_{\mathrm{ave} *}, n_{\min *}, n_{\max *}\right)$ are determined by the present day $(t=4.6 \mathrm{Gyr})$ solar wind conditions at $1 \mathrm{AU}$ (Earth orbit: $v_{\mathrm{sw}} \approx 400 \mathrm{~km} \mathrm{~s}^{-1} ; n_{\mathrm{sw}} \approx$ $6.5 \mathrm{~cm}^{-3}$ ) resulting in $n_{\min *}=4.6 \times 10^{3} \mathrm{~cm}^{-3}, n_{\text {ave } *}=$ $1.04 \times 10^{5} \mathrm{~cm}^{-3}, n_{\max *}=2.35 \times 10^{6} \mathrm{~cm}^{-3}$ and $v_{*}=3200$ $\mathrm{km} \mathrm{s}^{-1}$ for the nominal case. The time constant $\tau=2.56 \times$ $10^{-2}$ Gyr according to Newkirk Jr. (1980) and $t$ is the time in Gyr. Figure 2 shows the expected enhancement in solar wind density and velocity as function of age from present time back to about 3.9 Gyr ago.

The density scaling (Eqs. (5)-(7)) was derived from the measured mass loss rates from Wood et al. (2005), hence using $\dot{M}=4 \pi r^{2} m_{\mathrm{p}} n_{\mathrm{sw}} v_{\mathrm{sw}}$, with proton mass $m_{\mathrm{p}}$ together with Eqs. (4)-(6) and $r=1$ AU yields a similar evolution of the mass loss rate as indicated by the shaded area in Fig. 1, valid for main-sequence G-type stars with an age of at least 0.7 Gyr (Grießmeier et al., 2007; Lichtenegger et al., 2010).

\section{The Extreme Period: The First 500 Myr After the Sun's Arrival at the ZAMS}

The first $500 \mathrm{Myr}$ after the origin of a terrestrial planet and the evolution of its host star to the ZAMS are the most crucial period for the development of a biosphere. During this early evolutionary stage a star (including the young Sun) is more active at short wavelengths and plasma outflows can therefore remove a planet's atmosphere or initial water inventory. To understand how the atmospheres of terrestrial planets were affected by the young Sun it is of tremendous importance to gain more knowledge from astrophysical observations of magnetic properties, location of activity centers, radiation, plasma outflow (e.g., winds, CMEs), mass loss, and rotation periods, of very young solar proxies and stars with ages $<500 \mathrm{Myr}$.

\subsection{The magnetic field and plasma outflow of the early young Sun}

The rotation period of the young Sun was much faster ( $\sim$ few days) during the first $500 \mathrm{Myr}$ after the arrival at the ZAMS, therefore the activity and magnetic field configuration was most likely quite different from the current appearance. Star-spots on the young solar analog EK Dra which rotates with a period of $\sim 2.68$ days are observed to be formed everywhere; from the low latitudes up to polar regions (Strassmeier, 2009). The young Sun probably had the same structure of active regions. The high latitude Sun-spots could change the structure of coronal magnetic fields as well as the formation of prominences, which might lead to a predominant occurrence of flares and CMEs near the polar regions. The polar CMEs may propagate significantly above the ecliptical plane, which may cause more convenient conditions for planetary evolution. However, not much is known about solar magnetic dynamo during that time. It is not well constrained what the duration of activity cycles was, and also the butterfly diagram and the other laws of current solar activity just after the arrival of the Sun at the ZAMS. Therefore, the understanding of solar influence on planets requires further modeling of solar magnetic dynamos with all consequences in appearance of magnetic flux at the surface and in the possible structure of coronal magnetic field in the young Sun. This may be related to the fact that very young stars rotate fast and their active regions appear therefore at high latitudes. Furthermore, if the magnetic field becomes more akin to a global dipole, then the escape of the stellar plasma may be more inhibited (Wood et al., 2005). Thus, a possibly preferred appearance of CMEs at polar regions during the fast rotating young Sun may lead to the propagation of shocks and magnetic ropes and clouds off the ecliptic plane before $4 \mathrm{Gyr}$ ago, which might completely change the current picture of solar/stellarplanetary atmosphere-magnetosphere interaction processes (Zaqarashvili et al., 2011). All these questions need to be answered in order to understand the influence of the young Sun on Solar System dynamics after its arrival at the ZAMS.

\subsection{X-ray to UV fluxes after the Sun arrived at the ZAMS}

One can see from Table 1 that the "Sun in Time" sample contains 6 nearby G0 V-G5 V stars (EK Dra, $\pi^{1} \mathrm{UMa}$, HN Peg, $\chi^{1}$ Ori, BE Cet, $\kappa^{1}$ Cet) that have known rotation periods and well-determined temperatures, luminosities, and metallicities which fall into the early age domain where $t \leq 0.65$ Gyr after the Sun's arrival at the ZAMS.

Table 7 shows similar as Table 5 integrated radiation enhancement factors according to the analyzed data for various wavelength regions as a function of a stellar or solar age between $0.1-0.7$ Gyr. As in Table 5 the fluxes are divided in six wavelength intervals and are normalized to the present solar values, calculated by using the power laws as given in Section 3.1. The sums of observed emission line fluxes, are used as proxies for the evolution in the full UV range. The evolution of the integrated fluxes is displayed in Fig. 3. According to Ribas et al. (2005) the main uncertainties are the measurement error of the data, the calibration uncertainty of the various instruments and the intrinsic stellar variability which results in the total uncertainty of the power law slopes of $\leq 0.1$. The slope of the normalized fluxes becomes less steep with increasing wavelength, except for the 1300$1700 \AA$ interval. By comparing the data obtained from solar proxies with ages of about 4.6-0.7 Gyr (Table 5) with those which have ages $<0.7$ Gyr (Table 7 ) one can see that the upper atmospheres of early Venus, Earth, Mars and Saturn's satellite Titan should have been tremendously affected by X-rays, SXR and EUV radiation.

Short-wavelength radiation not only gets stronger toward younger solar analogs, it also becomes spectrally harder. This property is by no means trivial. In principle, increased luminosity could be achieved by populating the stellar surface with a larger number of similar magnetic active re- 
Table 7. Solar radiation flux enhancement as function of wavelength normalized to the present solar flux value during the first 500 Myr after the young Sun arrived at the ZAMS (Ribas et al., 2005; Güdel, 2007).

\begin{tabular}{|c|c|c|c|c|c|c|c|}
\hline $\begin{array}{l}\text { Solar age } \\
{[\mathrm{Gyr}]}\end{array}$ & $\begin{array}{l}t \text { b.p. } \\
{[\mathrm{Gyr}]}\end{array}$ & $\begin{array}{c}\text { X-ray } \\
{[1-20 \AA]}\end{array}$ & $\begin{array}{c}\text { SXR } \\
{[20-100 \AA]}\end{array}$ & $\begin{array}{c}\text { EUV } \\
{[100-920 \AA]}\end{array}$ & $\begin{array}{c}\text { FUV } \\
{[920-1180 \AA]}\end{array}$ & $\begin{array}{c}\text { Lyman- } \alpha \\
{[1200-1300 \AA]}\end{array}$ & $\begin{array}{c}\text { UV } \\
{\left[\begin{array}{ll}1300-1700 & \AA\end{array}\right]} \\
\end{array}$ \\
\hline 0.7 & 3.9 & 37 & 11 & 8.6 & 5 & 3.9 & - \\
\hline 0.65 & 3.95 & 43 & 12 & 9.4 & 5.3 & 4.1 & 5.8 \\
\hline 0.6 & 4.0 & 50 & 13 & 10 & 5.7 & 4.3 & - \\
\hline 0.55 & 4.05 & 59 & 15 & 11 & 6.1 & 4.6 & - \\
\hline 0.5 & 4.1 & 71 & 17 & 13 & 6.6 & 4.9 & - \\
\hline 0.45 & 4.15 & 87 & 19 & 14 & 7.2 & 5.3 & - \\
\hline 0.4 & 4.2 & 109 & 22 & 17 & 8 & 5.8 & - \\
\hline 0.35 & 4.25 & 141 & 26 & 19 & 9 & 6.4 & - \\
\hline 0.3 & 4.3 & 189 & 32 & 23 & 10 & 7.1 & 10.6 \\
\hline 0.25 & 4.35 & 268 & 40 & 28 & 12 & 8.1 & - \\
\hline 0.2 & 4.4 & 412 & 54 & 37 & 14 & 9.6 & - \\
\hline 0.15 & 4.45 & 715 & 77 & 51 & 18 & 11.8 & - \\
\hline 0.1 & 4.5 & 1558 & 129 & 82 & 26 & 15.8 & 40.5 \\
\hline
\end{tabular}

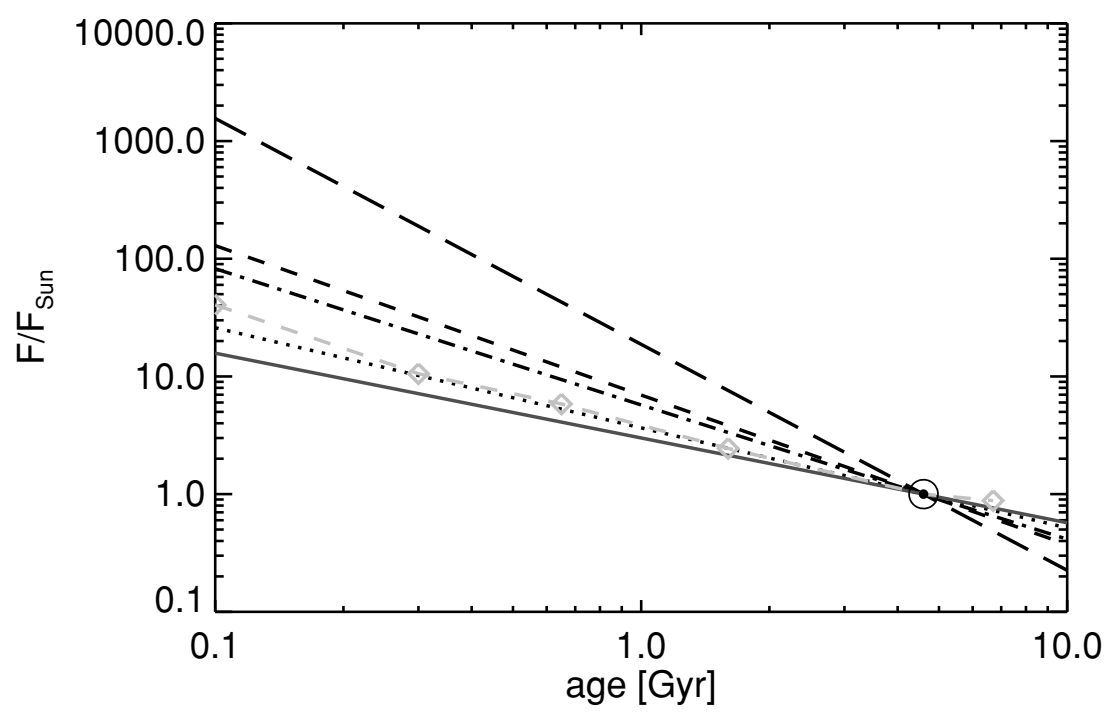

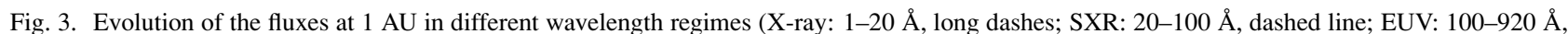

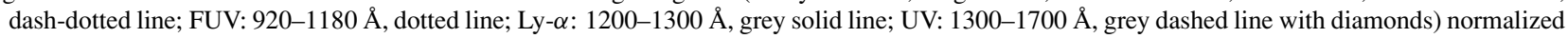
to the present solar values. Power laws for the wavelength ranges were taken from Ribas et al. (2005). Line fluxes in the UV (1300-1700 $\AA$ ) were summed up directly from observational data of strong emission lines (table 6 in Ribas et al., 2005).

gions, which would not imply a change in hardness.

Observationally, spectral hardness has been characterized in terms of the average electron temperature of the emitting stellar coronal plasma. While the solar coronal plasma shows characteristic temperatures in the 1-2 MK range (e.g., Peres et al., 2000), the overall trend for solar analogs with ages between $100 \mathrm{Myr}$ and 5 Gyrs can be given as,

$$
T_{\text {cor }}=0.34 L_{\mathrm{X}}^{0.25} \quad[\mathrm{~K}]
$$

where $T_{\text {cor }}$ is the average coronal temperature in $\mathrm{K}$ and $L_{\mathrm{X}}$ is the X-ray luminosity in $\mathrm{erg} \mathrm{s}^{-1}$ (Güdel et al., 1997; Güdel, 2007; Telleschi et al., 2005). For a young, near-ZAMS solar analog, $T_{\text {cor }} \approx 10 \mathrm{MK}$, which results in considerably harder continuous $\mathrm{X}$-ray radiation from these objects than from the present-day non-flaring Sun. This may be very important for modifying the photochemistry of early planetary atmospheres as the penetration depth is larger for high-energy (harder) photons.

The cause of the hardening with increasing luminosity is an increasing population of coronal loops with maximum temperature around $\sim 10 \mathrm{MK}$ with increasing rotation of the star (e.g. Sanz-Forcada et al., 2003). The nature of these loops is not clear in detail, but an attractive possibility is the operation of flares. For flares, a similar temperatureluminosity relation applies in X-rays (Güdel, 2004). Statistical studies during the past decade have addressed the problem of the occurrence rate of flares as a function of their total radiative energy (e.g., Audard et al., 2000; Güdel et al., 2003). The occurrence rate distributions are steep power-laws in energy. The summation of all small flares unresolved in the light curves may suffice to generate the entire observed radiation including "quiescent radiation". Toward more active stars, progressively larger flares contribute to this baseline level; because larger flares are hotter, the summed, average radiation also becomes harder. This 


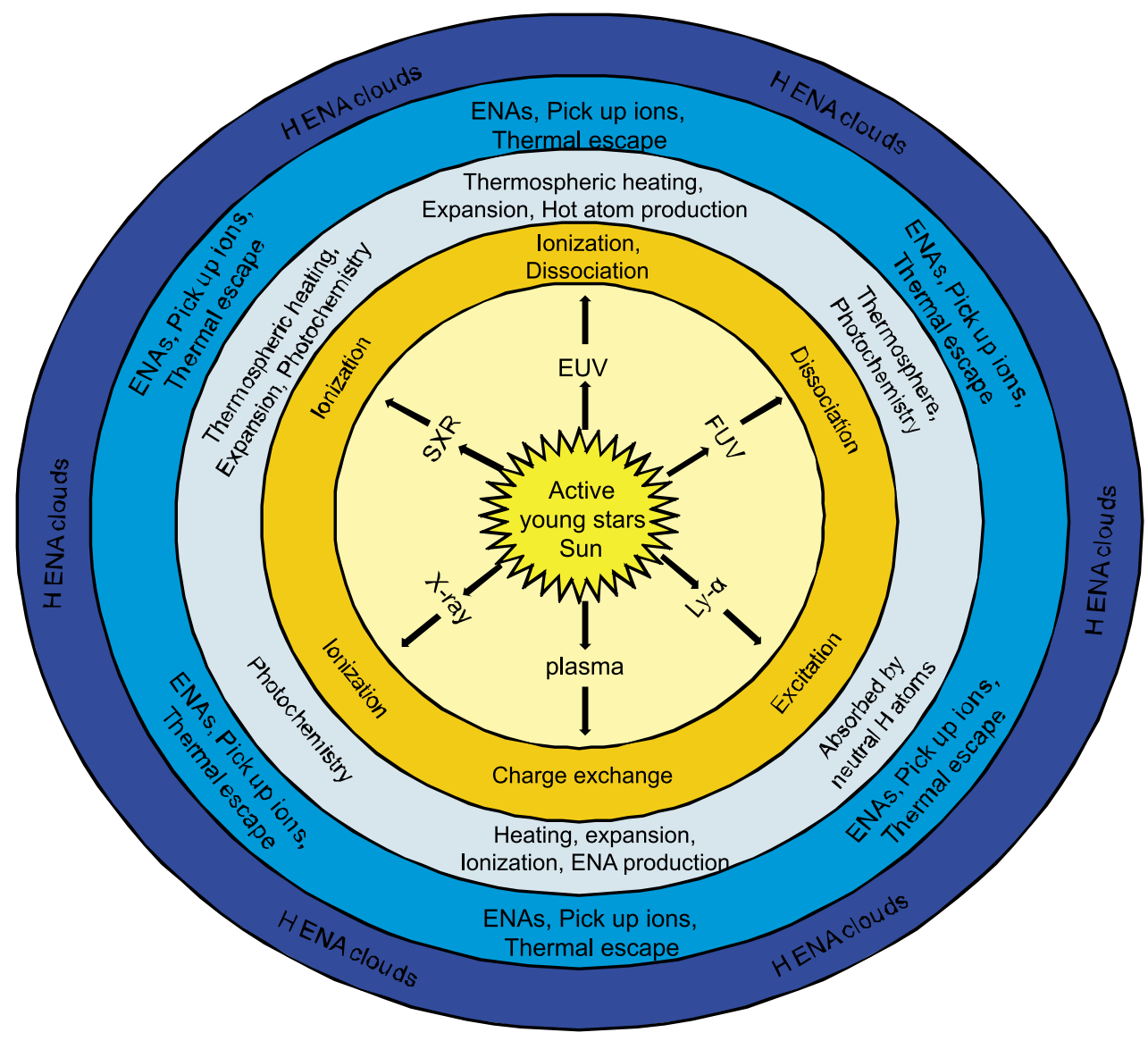

Fig. 4. Illustration of processes and response of upper atmospheres related to the radiation and plasma environment of active stars or the young Sun.

model is also supported by the continuous presence of nonthermal electrons in extremely active young solar analogs (Güdel, 2007) that are presumably accelerated by frequent flares. One would thus expect not only more intense Xrays in young solar analogs or around the young Sun itself, but also an increased level of flare-produced hard X-rays, gamma rays, ejected energetic particles and CMEs.

From this observational evidence one can expect that early planetary thermospheres should have been extremely hot and most likely expanded (Lammer et al., 2008; references therein). In Section 5 we will discuss the problems regarding the "survival" or stability of planetary atmospheres during such extreme solar/stellar activity periods.

\section{Solar Activity Response of Planetary Upper Atmospheres}

In recent studies by Kulikov et al. (2006, 2007), Lammer et al. (2006, 2008), Lundin et al. (2007), Tian et al. (2008a, b) and illustrated in Fig. 4 it was shown that enhanced solar EUV radiation and plasma (winds, CMEs) should result in permanent forcing of the upper planetary atmospheres, which can ionize, heat, expand, chemically modify, and erode it during the early phase of a planetary lifetime. In some cases the thermosphere may expand beyond an atmosphere protecting magnetospheric shield. Highly irradiated terrestrial planets can thus be even in danger of being stripped of their whole atmospheres (Lundin et al., 2007; Lammer et al., 2009; Lichtenegger et al., 2010).
When the temperature of the thermosphere is large, a significant fraction of the light constituents of the upper atmosphere attain velocities above the escape value, and the thermosphere may even become non-hydrostatic and start to expand accompanied by adiabatic cooling to several planetary radii (Chassefière, 1996; Tian et al., 2005, 2008a, b, 2009; Kulikov et al., 2007; Lammer et al., 2008).

On the basis of their energy source, we can separate two main escape categories: thermal escape and non-thermal escape. For thermal escape, atoms at the exobase level have more or less a Maxwellian velocity distribution that is determined by the exobase temperature. Particles with velocities which are larger than the escape velocity of the planet can escape from the planet (e.g., Chamberlain, 1963). In extreme cases when the mean thermal energy of the major species at the exobase level (where the mean free path of the particles is comparable to the atmospheric scale height) exceeds their gravitational potential energy, thermal escape can be considered as "blow-off" (Öpik, 1963). Assuming hydrostatic equilibrium, Kulikov et al. (2007) showed that terrestrial upper atmospheres exposed to enhanced EUV radiation of the early Sun could be heated to extremely high exobase temperatures which could be over $10000 \mathrm{~K}$. Tian et al. $(2008 \mathrm{a}, \mathrm{b})$ studied the response of thermospheres against high EUV fluxes in more detail and discovered that one can classify the thermosphere response into two thermal regimes.

In regime I, the thermosphere is in hydrostatic equilib- 


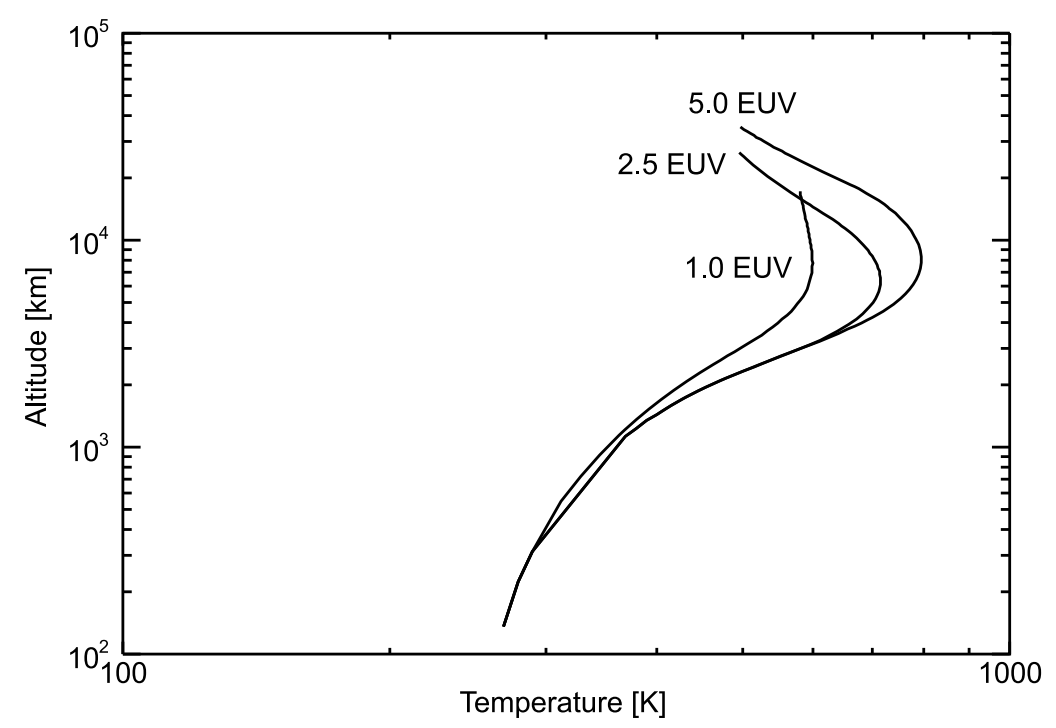

Fig. 5. Thermospheric temperature profiles for a H-rich upper atmosphere of an Earth-like planet between the mesopause and exobase level for present day EUV (1 EUV), 2.5 EUV and 5 EUV. According to Tian et al. (2005); for an EUV flux which is 5 times that of the present Sun the exobase moves upward to about 7 Earth radii.

rium in which the bulk atmosphere below the exobase can be considered as static. This is the situation in the thermospheres of the Solar System planets today. However, if the Sun's EUV flux or that of another star was/is much higher than that of the present Sun the thermosphere can enter a second regime.

In regime II the upper atmosphere becomes nonhydrostatic. In the hydrodynamic flow regime, the major gases in the thermosphere can expand very efficiently to several planetary radii above the planetary surface. As a result light atoms experience high thermal escape rates and expand to large distances (Tian et al., 2008a, 2009) where the whole atmosphere may be eroded by solar/stellar wind plasma flow related non-thermal escape processes (Khodachenko et al., 2007; Lammer et al., 2007; Lundin et al., 2007; Lichtenegger et al., 2010).

\subsection{Hydrogen-rich upper atmospheres of terrestrial planets}

Terrestrial planets may evolve through phases where their upper atmospheres are hydrogen-rich. There is some evidence from planetary formation theories that early Venus once lost its initial $\mathrm{H}_{2} \mathrm{O}$ inventory during an episode of a runaway and/or wet greenhouse effect via thermal and nonthermal atmospheric escape processes which could have been related to the form of "hydrodynamic" outflow. These extreme conditions may be reached in H-rich primordial thermospheres heated by the strong EUV flux of the young Sun (Sekiya et al., 1980a, b; Watson et al., 1981; Zahnle and Walker, 1982; Tian et al., 2005).

Sekiya et al. (1980a, b) and Watson et al. (1981) in their early works studied hydrodynamic conditions of $\mathrm{H}$ rich thermospheres from early Earth or Venus due to solar EUV heating by applying idealized hydrodynamic equations. From their thermospheric model Watson et al. (1981) obtained exobase levels for an Earth which is exposed to EUV fluxes of $\sim 5$ times that of the present Sun of $\sim 7$ planetary radii. This result also agrees with hydrodynamic model simulations for a H-rich early Earth thermosphere by Tian $e t$ al. (2005). An idea that EUV exposed upper atmospheres expand dynamically to larger distances is also confirmed by more sophisticated models such as that of Chassefière (1996), and Tian et al. (2008a, b, 2009) which matched subsonic outflow solutions to Jeans boundary conditions at the exobase.

Figure 5 shows the thermospheric temperature profiles between the mesopause and exobase altitudes for a $\mathrm{H}$-rich Earth-like planet which is exposed to 1 (present day), 2.5 and 5 times the present solar EUV flux value. One should keep in mind that we presented evidence in the previous sections that the EUV flux of the young Sun was much larger than 5 times that of the present Sun during the first 700 Myr after the Sun arrived at the ZAMS (see Table 7). During such stellar activity periods hydrogen-rich terrestrial planets may appear more as small gas giants with upper atmospheres which expand several tens of planetary radii.

\subsection{Response of $\mathrm{N}_{2}$-rich atmospheres to high EUV fluxes}

Tian et al. (2008a, b) developed an advanced multicomponent, hydrodynamic thermosphere model to study self-consistently the response of the Earth's thermosphere under EUV flux values from 1 (present) to 20 times that of the present-days solar value by assuming a present-time Earth atmospheric composition. Their model is validated against observations (MSIS-00: Hedin, 1991) and in agreement with other thermospheric models (e.g., Roble et al., 1987; Smithtro and Sojka, 2005a, b) applied to the present day Earth. Figure 6 shows the thermospheric temperature profiles as a function of solar/stellar EUV flux values up to the corresponding exobase levels for various EUV cases. The results of these studies indicate that the thermosphere of Earth or a similar exoplanet with a present day Earth-like atmospheric composition should have switched from hydrostatic regime I to the non-hydrostatic regime II, if the thermospheric temperatures exceeded a critical value of about 


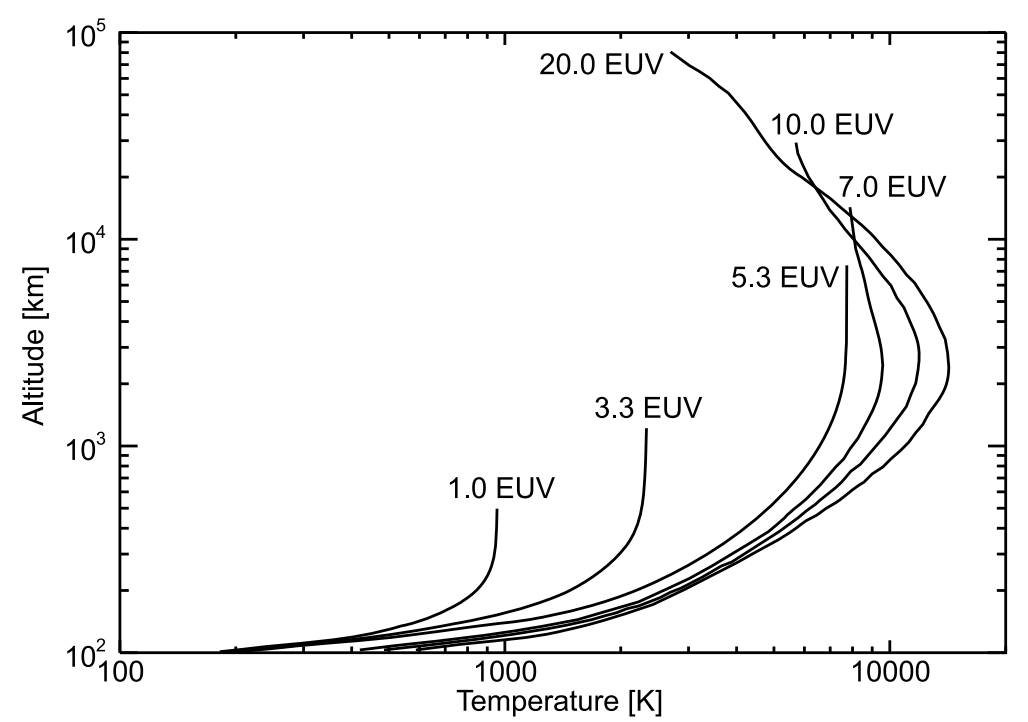

Fig. 6. Thermospheric temperature profiles between $100 \mathrm{~km}$ and the corresponding exobase levels for 1 (present), 3.3, 5.3, 7, 10 and 20 times higher EUV solar fluxes than today, applied to Earth with the present time atmospheric composition (Tian et al., 2008a; Lammer et al., 2008). One can see that the thermosphere switches in a non-hydrostatic regime for $T \geq 8000 \mathrm{~K}$ and expands dynamically up to distances beyond 10 planetary radii.

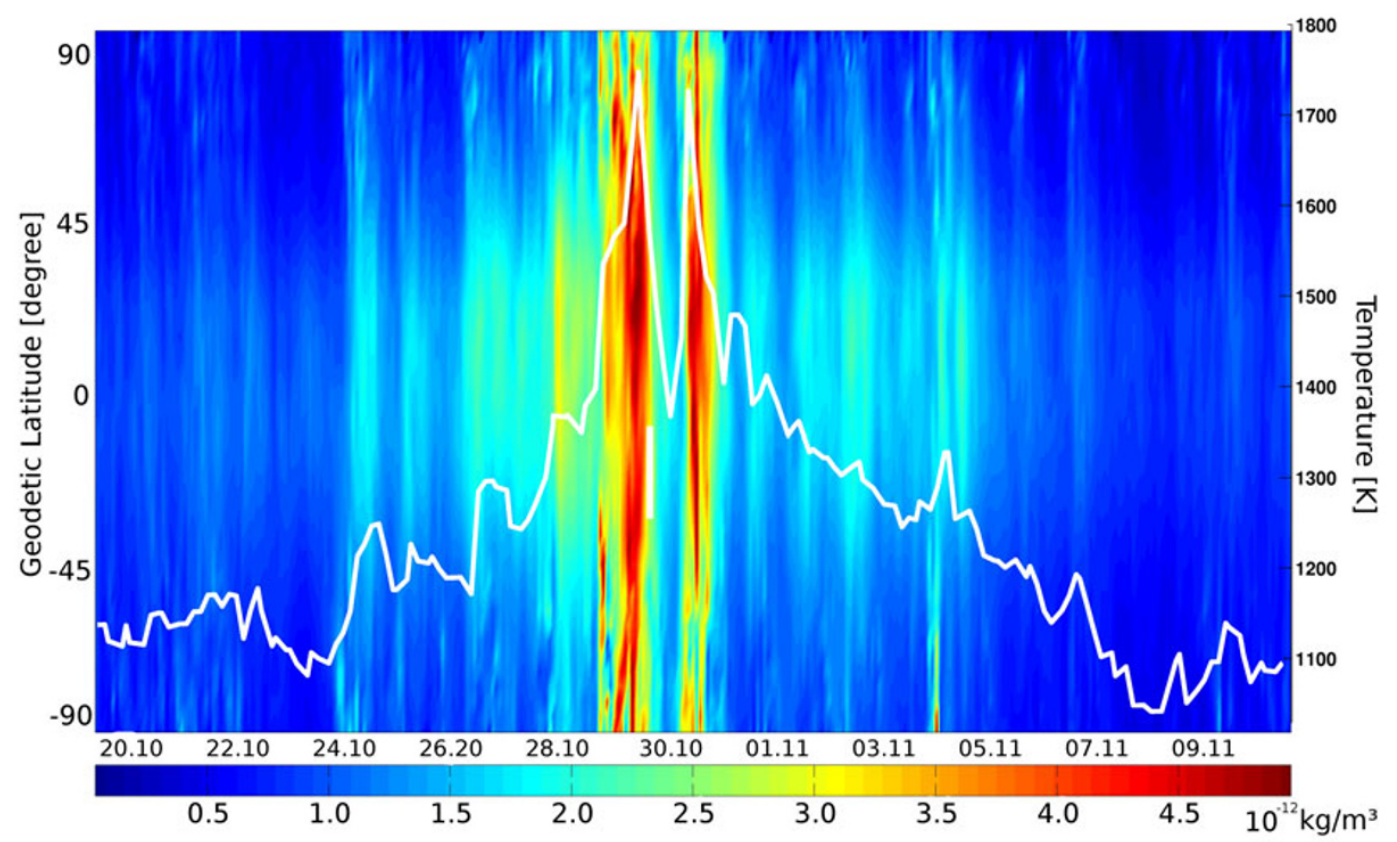

Fig. 7. Density impact of a X17.2 flare (Oct. 2003) observed by the GRACE satellite and temperature variation at orbit height is represented by the white curve (measurements taken at a local time of 16:19).

8000 K. For the Earth, these critical temperatures correspond to solar/stellar EUV flux values $\geq 6$ times that of the present Sun.

One can also see that the exobase distance which separates the collision dominated thermosphere from the collisionless exosphere moves from $\sim 500 \mathrm{~km}$ up to $\sim 12.7$ Earth-radii above the surface for a $20 \mathrm{EUV}$ (4.2 Gyr ago) solar flux which would move about $\sim 3.7$ Earth-radii above the present-day average subsolar magnetopause location of $\sim 9$ Earth-radii. Under such extreme SXR and EUV conditions the magnetosphere would not protect the upper atmosphere, the planet experiences Venus or Mars-like solar wind-atmospheric plasma interaction and as a result the exosphere could experience strong non-thermal escape.

The conjecture that the upper atmosphere of the Earth responds strongly to the solar X-ray, SXR and EUV radiation and particle fluxes can also be seen and studied from density enhancements during extreme solar events which are observed by low Earth orbit satellites (e.g., Sutton et al., 2006; Shematovich et al., 2011). Figure 7 shows the atmospheric neutral densities determined from accelerometer measurements onboard the low Earth orbiter Gravity Recovery And Climate Experiment (GRACE) satellite, where the neutral densities are determined with a 60 second resolution at an 


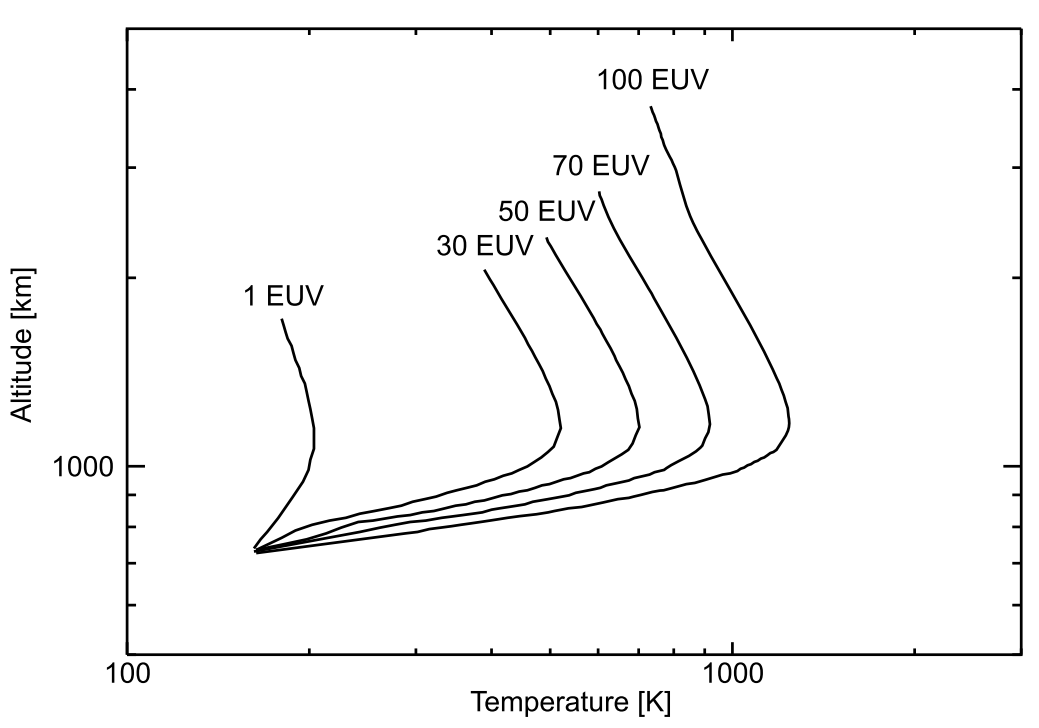

Fig. 8. Thermospheric temperature profiles between the mesopause and the corresponding exobase levels for 1 (present), 30, 50, 70,100 times higher EUV solar fluxes than today, expected at Saturn's large satellite Titan with the present time atmospheric composition.

orbital distance between $470 \mathrm{~km}$ at the perigee and $520 \mathrm{~km}$ at the apogee. The period under investigation covered ten days before and after the so-called Halloween geomagnetic storm (X17.2 flare) in 2003 (Sutton et al., 2006). During this particular extreme solar event the upper atmosphere of the Earth was heated and as a result expanded with a density increase at the satellites orbit of $\approx 400 \%$ compared to that at quiet conditions (Shematovich et al., 2011). Related to that, also the temperature at the satellite position, calculated using the "Jacchia-Bowman model" (Bowman et al., 2008 ), increases significantly from $\sim 900 \mathrm{~K}$ up to $\sim 1800$ $\mathrm{K}$. By applying a test-particle model to the expanded thermospheres given in Tian et al. (2008a) and a young solar wind which was about 100 times denser and twice as fast as today one obtains $\mathrm{N}^{+}$ion pick up loss rates in the orders of $\sim 10^{29}-10^{30} \mathrm{~s}^{-1}$ (Lichtenegger et al., 2010). Due to the extended thermosphere-exosphere region the Earth's entire current $\mathrm{N}_{2}$ atmosphere ( $\sim 700$ mbar) could have been stripped within a few million years.

In view of large uncertainties in the solar wind evolution discussed in Section 3.3 Lichtenegger et al. (2010) estimated also the ion pick up loss rate of these $\mathrm{N}_{2}$ atmospheres by assuming present-day solar wind parameters. Although the loss rates are about a factor 10 lower, Earth would have lost its present nitrogen atmosphere within some tens of million years at the latest when the EUV flux was $>10$ times from that of the present Sun. The main reason for these large escape rates is the EUV heated and expanded large solar wind plasma interaction area which is not protected beyond a present Earth-type magnetospheric shield.

Because the Earth's nitrogen inventory is not lost, Lichtenegger et al. (2010) argued that these results indicate that the $\mathrm{CO}_{2}$ amount in the early $\mathrm{N}_{2}$-rich terrestrial atmosphere was at least two orders of magnitude higher than the present-time level. Such an amount was needed to confine the upper atmosphere after the onset of the geodynamo within the shielding magnetosphere and thus might have protected it from complete destruction.
However, the model simulation results show that planets with the size and mass $\leq$ of the Earth with even a $95 \% \mathrm{CO}_{2}$ atmosphere would experience problems when the EUV flux reaches values which are $\geq 30$ time that of the present Earth (Tian, 2009; Lichtenegger et al., 2010). Moreover, photochemically produced low energy ("hot") atoms and additional thermospheric heating due to energetic solar/stellar particles could have additionally enhanced the atmospheric escape. On the other hand the expected initial $\mathrm{CO}_{2}$ reservoir on early Earth was perhaps much larger compared to its nitrogen reservoir, so that a non-thermal loss to space of several bars of $\mathrm{CO}_{2}$ during the first 100-300 Myr would have only slightly modified the $\mathrm{CO}_{2}$ budget of the Earth.

Figure 8 shows the expansion of the second $\mathrm{N}_{2}$-type atmosphere of a solar system body, namely Saturn's large satellite Titan. As pointed out by Lammer et al. (2008) self consistent hydrodynamic models applied to Titan's thermosphere would be needed to study the photochemistry changes due to the different solar flux in the past to examine the modification in thermospheric temperature. Such studies are important for investigating to which distance the exobase could expand due to EUV heating by the young Sun and if Titan's upper atmosphere could have reached classical hydrodynamic "blow-off" conditions or not.

\subsection{Response of $\mathrm{CO}_{2}$-rich atmospheres to high EUV fluxes}

High amounts of $\mathrm{CO}_{2}$, like on present Venus, Mars, and most likely on early Earth before 4 Gyr ago can cool the thermosphere much better than relatively dry Earth-like $\mathrm{N}_{2} / \mathrm{O}_{2}$-type atmospheres, so that the exobase level remains closer to the planet's surface and its upper atmosphere is better protected against erosion by the solar or stellar wind. For the more moderate EUV-flux period non-thermally photochemically produced suprathermal "hot" O atoms populate the exospheres of Venus and Mars. Gröller et al. (2010) developed a sophisticated Monte Carlo model and applied it recently to Venus during low and high solar EUV activity. One can see from Fig. 9 that the hot $\mathrm{O}$ atom corona densi- 


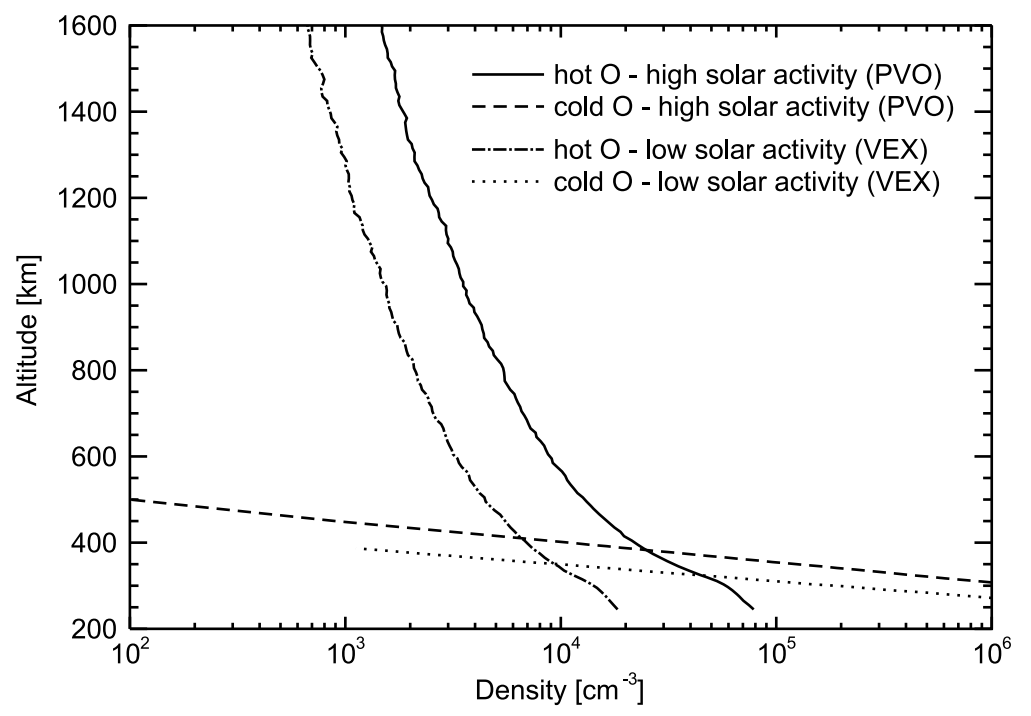

Fig. 9. Photochemically produced supra-thermal "hot" O atom density profiles at present Venus during low and high solar activity conditions compared with the bulk atmosphere.

ties for low solar activity (VEX solar conditions) are about a factor of 2-3 smaller than for high solar activity. Nonthermal hot $\mathrm{O}$ atoms populate the environment around the planet up to large distances where they can interact with the solar wind. This behavior indicates that higher EUV fluxes during the young Sun periods should have also resulted in denser hot $\mathrm{O}$ coronae around Venus and Mars, resulting in higher pick up loss rates.

Kulikov et al. (2006) studied the expected $\mathrm{O}^{+}$ion pick up loss rates over Venus' history by using EUV data from solar proxies discussed in Section 2 and a range of solar wind plasma densities and velocities which were expected for the young active Sun. These authors used a diffusivegravitational equilibrium and thermal balance model, which was applied for a study of the heating of the early thermosphere by photodissociation and ionization processes, exothermic chemical reactions, and cooling by $\mathrm{CO}_{2}$ IR emission in the $15 \mu \mathrm{m}$ band. The results of their study yield expanded thermospheres with exobase altitudes between about $200 \mathrm{~km}$ for present EUV flux values and about $1700 \mathrm{~km}$ for 100 times higher EUV fluxes after the Sun arrived at the ZAMS. The exobase temperatures yield values of about $8000 \mathrm{~K}$ for the earliest EUV fluxes after the Sun arrived at the ZAMS.

However, one can expect that the high EUV flux will dissociate $\mathrm{CO}_{2}$ molecules in the thermosphere which results in less IR-cooling and in a possible non-hydrostatic upper atmosphere environment with high thermal escape rates for dissociated $\mathrm{C}$ and $\mathrm{O}$ atoms. By considering the uncertainties discussed on the solar wind parameters expected for the young Sun as discussed in Section 3.3, with the model discussed in Kulikov et al. (2006) one obtains loss rate from a $\mathrm{CO}_{2}$-rich early Venus atmosphere which is equivalent to about 2-10 bars during the planets' history. From the present knowledge of the stellar wind parameters discussed in this work we expect that the high mass loss cases shown in Kulikov et al. (2006), where Venus could have lost much more atmosphere than 10 bar over its livetime may be over- estimations based on solar wind parameters which are too large.

If Venus evaporated its initial $\mathrm{H}_{2} \mathrm{O}$ inventory during that period, the planets upper atmosphere would have been hydrogen-rich (Chassefière, 1996) as discussed in Section 5.1 and a dense hydrogen corona would have protected the initial $\mathrm{N}_{2}$ and $\mathrm{CO}_{2}$ inventory from escape until the evaporated water inventory was lost by thermal and nonthermal escape processes.

Tian et al. (2009) and Tian (2009) applied an advanced 1-D multi-component, hydrodynamic, planetary thermosphere-ionosphere model and a coupled electron transport-energy deposition model to Mars and $\mathrm{CO}_{2}$-rich atmospheres of "super-Earths". With this model they could self-consistently calculate the ionization and excitation rates of thermospheric species together with the connected electron heating rates and related EUV heating efficiencies. It was found that due to the high EUV fluxes ( $>10$ times that of the present Sun) $\mathrm{CO}_{2}$ molecules can be dissociated so that less thermospheric IR-coolers are available which resulted in higher heating efficiencies and exobase temperatures compared to previous model simulations (Lammer et al., 2006; Kulikov et al., 2006). When extrapolating the model to early Mars, Tian et al. (2009) found that the exobase expands to $\sim 900 \mathrm{~km}$ altitude and the exobase temperature reaches $\sim 800 \mathrm{~K}$ for EUV flux values which are about 10 times the present solar value. As one can see from Fig. 10, for higher EUV fluxes such as 20 times the present solar value the exobase moved to distances of $\geq 10000 \mathrm{~km}$ with exobase temperatures $>2500 \mathrm{~K}$. One main reason for the nonlinear response of the upper atmosphere to the EUV flux is that the main IR cooling agents such as $\mathrm{CO}_{2}$, are destroyed by the high SXR and EUV fluxes, so that thermospheric cooling becomes less efficient.

According to their results the thermal neutral atom $\mathrm{C}$ and $\mathrm{O}$ escape fluxes $>10^{11} \mathrm{~cm} \mathrm{~s}^{-1}$ from early Mars were as high as the outgassing fluxes which depended on ini- 


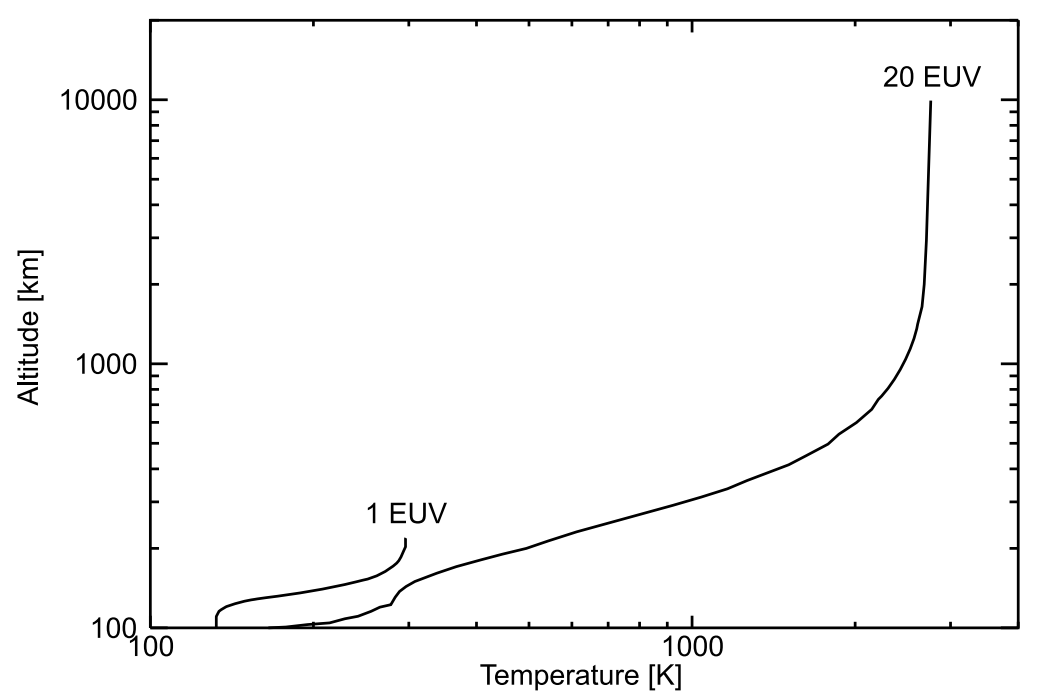

Fig. 10. Thermospheric temperature profiles for present day Mars between $100 \mathrm{~km}$ and the corresponding exobase levels for present time solar EUV flux values (1) (Fox and Hać, 2009) and the martian thermosphere when it is exposed to a 20 times higher EUV flux ( 4 Gyr ago according to Tian et al. (2009)). The thermosphere temperature reaches $\sim 3000 \mathrm{~K}$ and expands dynamically upward to distances of about 3 planetary radii.

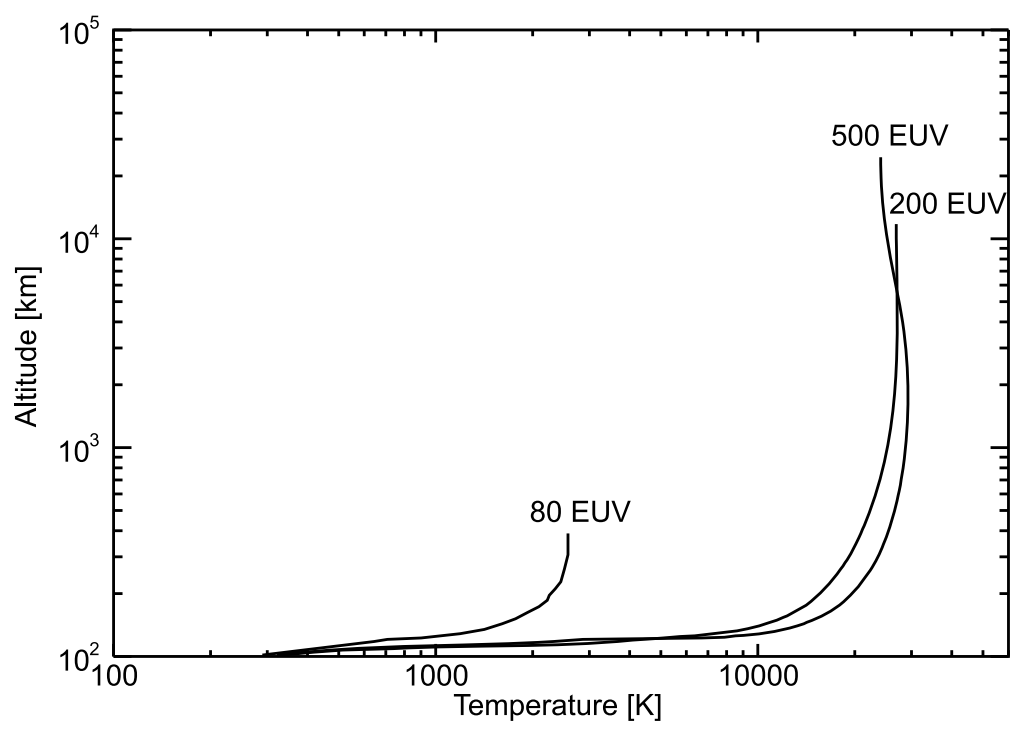

Fig. 11. Thermospheric temperature profiles according to Tian (2009) for "super-Earths" with $\sim 6$ Earth masses and $\mathrm{CO}_{2}$-type atmospheres within orbits of M-star habitable zones between $100 \mathrm{~km}$ and the corresponding exobase levels when their thermospheres exposed to a 80, 200 and 800 times higher EUV flux.

tial $\mathrm{CO}_{2}$ inventories (e.g. Phillips et al., 2001) during the early Noachian when the EUV flux was even larger than 20 times of the solar value. Due to the EUV heating and expansion of the upper atmosphere an early martian magnetic field could have not most likely protect the ion escape of $\mathrm{C}^{+}$ and $\mathrm{O}^{+}$atoms which originated from dissociation of $\mathrm{CO}_{2}$ molecules. By applying the same test-particle model, as in the study of early Earth by Lichtenegger et al. (2010), to the upper atmosphere profiles and estimated martian magnetopause distances as given in Dehant et al. (2007), we can show that the expanded thermosphere-exosphere can interact with the solar wind plasma which results in pick up $\mathrm{C}^{+}$ ion escape fluxes of $>10^{11} \mathrm{~cm} \mathrm{~s}^{-1}$ before $4 \mathrm{Gyr}$ ago.

Thus, due to the high activity of young Sun and the low gravity of Mars a dense early Noachian martian atmosphere may not have built up during the first 300-400 Myr after the planet's origin. A question remains when the outgassing rate was larger compared to the thermal and non-thermal neutral escape rates and what role impacts play in the formation or removal of the atmosphere (Pham et al., 2009). These processes may have played a role at the end of the Noachian so that a $\mathrm{CO}_{2}$ atmosphere with a surface pressure of a few 100 mbars and a resulting warmer climate may have been established so that Mars warmed up sporadically $\sim 3.5-4$ Gyr ago. However, such an accumulated $\mathrm{CO}_{2}$ atmosphere should have been lost since about $4 \mathrm{Gyr}$ ago by solar wind erosion (ion pick up, cold ion outflow), sputtering and photochemical hot atom escape after the martian magnetic dynamo stopped working.

Tian (2009) applied the same model to more massive $\sim 6$ 


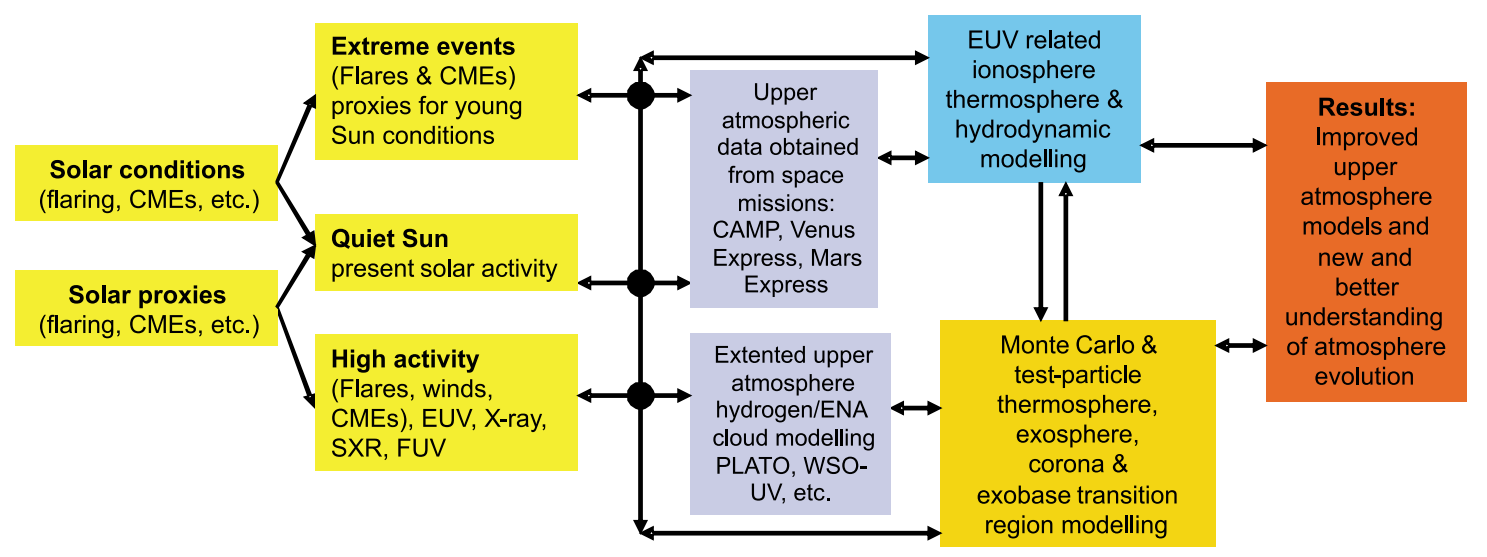

Fig. 12. Illustration chart about the general approach and connections between observations and modeling efforts which should lead to the development of state-of-the-art thermosphere-exosphere models and to a better understanding of aeronomically related atmospheric evolution aspects for terrestrial planets.

Earth-mass hypothetical $\mathrm{CO}_{2}$-rich exoplanets. The results indicate that such atmospheres are stable against thermal escape, even for M stars' EUV flux enhancements as large as 1000 compared to the present Sun. On the other hand one can see from Fig. 11 that the highly irradiated thermospheres of such planets may also expand to altitudes beyond $10000 \mathrm{~km}$ above the planets surface. These results are in agreement with previous studies of Khodachenko et al. (2007) and Lammer et al. (2007) who estimated the atmospheric erosion of $\mathrm{CO}_{2}$-rich exoplanets due to CMEinduced ion pick up within close-in habitable zones of active M-type dwarf stars. They found that Earth-mass $\mathrm{CO}_{2}-$ rich exoplanets that have no, or weak, magnetic moments may lose tens to hundreds of bars of atmospheric pressure, or even be stripped of their whole atmospheres due to CMEinduced $\mathrm{O}^{+}$ion pick up at orbital distances $\sim 0.2 \mathrm{AU}$. Although the formation of magnetospheres and possible locations of a magnetopause are not well known for such planets, expanded atmospheres of "super-Earths" could have been subject to stellar wind erosion. However, one should note that previous studies focused either on thermal effects and escape (e.g., Tian et al., 2008a, 2009; Tian, 2009) or on non-thermal processes (e.g., Kulikov et al., 2006, 2007; Terada et al., 2009; Lichtenegger et al., 2010) although both processes are strongly coupled and should not be separately studied. As illustrated in Fig. 12, to understand really how planetary atmospheres can "survive" the extreme X-ray, SXR and EUV activity of their host stars after they arrived at the ZAMS, one has to combine thermal and non-thermal upper atmosphere processes by developing a self-consistent multi-species ionospheric-thermospheric exosphere model which couples thermal and non-thermal processes, for studying hydrostatic and non-hydrostatic dynamically expanding thermosphere-exosphere regions, including the investigation of the production of hot atoms, their collisional interaction and transport within highly EUV exposed thermosphere-exosphere regions, and their contribution to the thermospheric heat budget.

\section{Testing Atmosphere Evolution Scenarios via UV-transit Observations of Earth-like Exo- planets Orbiting Dwarf Stars}

As discussed in the previous section, dynamically expanded thermospheres and related coronae should form if SXR and EUV fluxes $\geq 5$ EUV and light atoms such as hydrogen populate the upper atmosphere, or if no IR-cooling molecules are present. As a consequence the upper atmosphere expands beyond a possible magnetospheric shield and hydrogen energetic neutral atoms (ENAs) are produced via charge exchange collisions between the atmosphere and charged solar/stellar wind particles (Lammer et al., 2011a, b). As discussed recently by Holmström et al. (2008), Ekenbäck et al. (2010) and Lammer et al. (2011a) ENAs have been observed around all Solar System planets when a spacecraft was equipped with an accurate instrument.

Lammer et al. (2011b) pointed out that, the observation and theoretical modeling of hydrogen and ENA-clouds around a terrestrial exoplanet would indicate that its thermosphere is not in hydrostatic equilibrium but experiences hydrodynamic expansion beyond its magnetospheric shield. Therefore, by observing planetary hydrogen clouds and related ENA-coronae around transiting terrestrial exoplanets in UV (Lyman- $\alpha$ attenuation) we will learn more on how the early Solar System planetary atmospheres have evolved. Because transiting Earth-size exoplanets and their hydrogen clouds are too small relative to the size of Sun-like G-stars to detect them, Lammer et al. (2011b) proposed to study hydrogen clouds around such planets within orbits of $\mathrm{M}$ dwarf stars. Due to the large number of M-type stars in our neighborhood (Scalo et al., 2007) and their small size and comparably long periods of strong stellar activity in X-rays, SXR, and EUV M dwarf stars may represent the most promising candidates for the detection of hydrogen clouds and related ENAs.

Terrestrial planets such as the Earth are formed via collisions between planetary embryos and by accumulation of planetesimals in the presence of the primordial solar/stellar nebula gases (e.g. Alibert et al., 2010; references therein). At this early formation stage young terrestrial planets are surrounded by massive primordial gas envelopes which are 


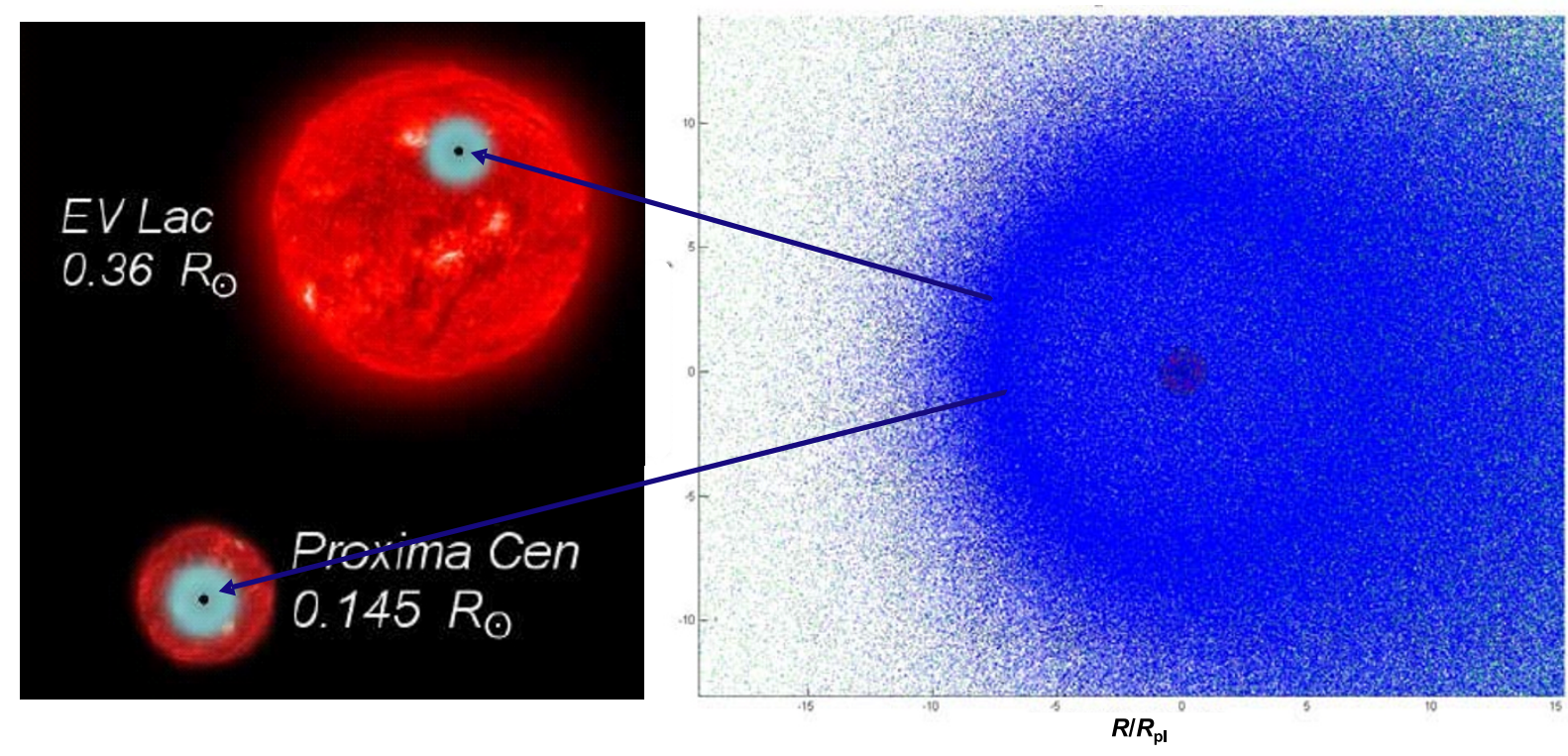

Fig. 13. Left side illustrates the size relation of exoplanets around the two M-stars EV Lacertae and Proxima Centauri with a transiting Earth analogue with an extended hydrogen atmosphere of $7 R_{\text {Earth }}$. Right side shows a preliminary model simulation of an extended hydrogen corona and stellar wind produced hydrogen ENAs around an Earth-size planet with an expanded hydrogen-rich thermosphere of $7 R_{\text {Earth }}$ (Lammer et al., 2011b).

mainly composed of hydrogen and helium. According to Lammer et al. (2011b) such examples may be:

- dense hydrogen envelopes which remained from the primordial nebulae;

- hydrogen-rich thermospheres produced via dissociation of $\mathrm{CH}_{4}, \mathrm{NH}_{3}$ and $\mathrm{H}_{2} \mathrm{O}$ molecules;

- ocean planets or planets which evaporate their initial $\mathrm{H}_{2} \mathrm{O}$ reservoir;

- evaporation of $\mathrm{H}_{2} \mathrm{O}$ oceans due to cometary or asteroidal impacts.

During this early stage in planetary evolution, heavy atmospheric species such as $\mathrm{CO}_{2}, \mathrm{~N}_{2}$, etc. are most likely protected from escape to space until the dense primordial hydrogen envelope which populates the non-hydrostatic thermosphere is lost. The removal time of such hydrogen envelopes depends on the amount of available hydrogen, the SXR, EUV flux and plasma environment of the young host star. If this hypothesis is right, one can expect that hydrogen-rich upper atmospheres may be a common scenario at young terrestrial planets during tens or hundreds of Myr after their origin.

If we expect that similar processes produce hydrogenrich thermospheres around many young terrestrial exoplanets, these bodies would produce huge planetary hydrogen coronae and related ENA clouds as observed around hot gas giants such as HD 209458b (Vidal-Madjar et al., 2003; Holmström et al., 2008; Ekenbäck et al., 2010) and HD 189733b (Lecavelier des Etangs et al., 2010). Figure 13 shows an illustration of such transiting exoplanets and a modeled hydrogen cloud around an Earth-size test planet accordingly to Lammer et al. (2011b). As one can see from Fig. 13 the produced hydrogen atoms populate a wide area around the planetary environment and a huge fraction of the host star should be covered with the outward flowing plan- etary hydrogen atoms and produced ENA-cloud during a transit.

Since the past years the search for Earth-like exoplanets around low-mass M-type stars has become one of the major activities in stellar astrophysics. Besides several groundbased search projects such as CRIRES, CARMENES, or HZPF (e.g., Lammer et al., 2011b; and references therein) ESA's M3 candidate PLATO (PLAnetary Transits and Oscillations of stars) will also be dedicated to detect transiting terrestrial exoplanets within and outside of habitable zones, and determine their basic parameters with unprecedented accuracy (Catala and the PLATO team, 2009a, b). The PLATO mission has been selected for a definition study phase in the context of ESA's Cosmic Vision 2015-2025 program as an M-class mission with foreseen launch in 2017-2024 (Stankov et al., 2010). The instrument concept of PLATO (Catala and the PLATO team, 2009a) consists of a multi-telescope approach. In total 32 refractive telescopes (pupil size about $120 \mathrm{~mm}$ each) are combined to produce highly photometrically accurate lightcurves covering a wide dynamic range. In addition, two so-called fast telescopes with fixed color filters and higher read-out cadence are used for seismic studies of the brightest targets. PLATO is planned to be launched into an orbit around the L2 Lagrange point. Two target fields will be monitored for at least 2-3 years with high duty cycle. In a "step-and-stare" phase several fields will be monitored for shorter durations (2-5 months each). In total, PLATO will cover about 50\% of the sky.

If selected, this mission will follow the ongoing CoRoT and Kepler missions to obtain a new sample of transiting exoplanets, focussing on terrestrial planets around bright stars $\left(m_{\mathrm{V}} \leq 11 \mathrm{mag}\right)$. This sample will not only provide planetary systems with highly accurately known basic planetary and stellar parameters, but will also provide access 
to a well-constrained age of planetary systems. Planetary radii will be determined from PLATO transit observations. Combined with a world-wide ground-based follow-up campaign to obtain radial velocity measurements, planet masses will be derived. However, previous transit searches have shown that well constrained stellar parameters are crucial to derive accurate planet parameters. PLATO therefore combines photometric transit search with astroseimology of the central stars of planetary systems. These astroseismological studies are further keys to obtain a well constrained age (goal: 10\% accuracy; Kjeldsen et al., 2009) of stars, and therefore planetary systems.

The search will include planets of all orbital periods and with all physical sizes, with focus on bright central stars of solar type. PLATO will perform a full characterization of the observed very bright stars using seismic analysis. The highest priority sample, therefore, are about 20000 dwarfs and sub-giants with $m_{\mathrm{V}} \leq 11$ mag for which Earth-sized planets can be detected and accurate seismic analysis be performed. A smaller sample of very bright stars $\left(m_{\mathrm{V}} \leq\right.$ $8 \mathrm{mag}$ ) will provide targets for future spectral characterization of the detected planets. Finally, a large sample of stars (more than 245000 cool dwarfs/subgiants with $11 \mathrm{mag}<$ $m_{\mathrm{V}}<13 \mathrm{mag}$ ) will be monitored to detect terrestrial planets, but without detailed astroseismology. What is interesting in the context of our work outlined in this section, PLATO will also search for exoplanets around more than 10000 nearby M-type dwarfs (down to $m_{\mathrm{V}}=15-16 \mathrm{mag}$ ), including planets within their habitable zones.

After the discovery of Earth-size exoplanets with PLATO, follow-up observations with the the upcoming World Space Observatory-UltraViolet (WSO-UV) mission could be carried out. The WSO-UV is an international space mission devoted to UV spectroscopy and imaging which is included in the Federal Space Program of the Russian Federation with a launch date around 2014-2015 (Shustov et al., 2009; Lammer et al., 2010). The observatory includes a $170 \mathrm{~cm}$ aperture telescope capable of highresolution and long slit low-resolution spectroscopy, and deep UV and optical imaging and is designed for observations in the UV-range (Shustov et al., 2009). The WSO-UV space observatory will be equipped with instruments which can carry out:

- High resolution spectroscopy of point sources in a wavelength range 1020-3200 $\AA$ by means of 2 high resolution echelle set ups with a sensitivity which is about 10 times better than that of the HST/STIS.

- Long-slit resolution spectroscopy in the 1020-3200 $\AA$ range.

- High resolution imaging in the 1500-2800 $\AA$ range with a field view of $1^{\prime} \times 1^{\prime}$.

- High resolution imaging in the 2000-7000 $\AA$ range with a sensitivity which is about 3 times higher than the HST/ACS system.

- Molecular hydrogen mapping in extended, faint astrophysical objects with a large field far UV (1150 $1900 \AA$ A) camera.

The WSO-UV's core program will focus also on exoplanetary atmospheres and astrochemistry in the presence of strong UV radiation fields (Gómez de Castro et al., 2009; Shustov et al., 2009). In Lammer et al. (2011b) it was shown that it may be possible to observe transits in UV of Earth-size exoplanets with extended hydrogen coronae if they orbit around small stars.

Thus, transit follow-up observations of exoplanets discovered by ESA's currently studied PLATO mission in the UV-range of terrestrial exoplanets around M-type stars with space observatories such as the WSO-UV would provide a unique opportunity to shed more light on the early evolution of Earth-like planets, including those of our own Solar System. Moreover, as pointed out in Lammer et al. (2011a) observations of exoplanets with various ages, together with hydrogen cloud and ENA modeling efforts could be used for the reconstruction of the solar wind history as well as testing magnetic dynamo evolution scenarios of gas giants.

\section{Conclusions}

By analyzing solar and astrophysical observations and phenomena we present strong evidence that the Sun and similar stars underwent much more active phases after they arrived at the ZAMS. The enhanced activity reveals itself in the form of strong high energy radiation emissions, frequent flares, and a denser and faster stellar wind. Results from the "Sun in Time" project suggest that the coronal X-ray and EUV emissions of the very young main-sequence Sun were $\sim 1550$ and $\sim 80$ times stronger than those of the present Sun, respectively. We showed that such an energy and particle environment certainly had an impact on the origin and evolution of Solar System planets and their atmospheres. Due to the heating of the much higher solar/stellar SXR and EUV flux the planetary thermospheres and exobase levels extended to higher altitudes than at present time, which resulted in larger solar wind-atmosphere interaction areas, no protection of intrinsic magnetospheres, the production of huge hydrogen ENA-clouds and higher non-thermal escape rates. Under certain activity conditions of the young Sun or planetary host star hydrostatic equilibrium could not have been kept. Finally we addressed the possibility of studying the detection and follow-up observations of extended upper atmospheres around exoplanets by near future space observatories such as ESAs PLATO mission in combination with the WSO-UV space telescope. Such observations would enhance our knowledge regarding the interaction of the host star's plasma environment and with Earth-like exoplanets itself, as well as shed light into the evolutionary stage of their atmospheres.

Acknowledgments. This research is supported by the Helmholtz Association through the research alliance "Planetary Evolution and Life." The authors also acknowledge support from the European Science Foundation (ESF) and the Europlanet Networking activity Na1 (Observational Infrastructure Networking), and Science Networking Na2 working group (WG4 and WG5) activities. The authors also acknowledge support from the Austrian FWF (Wissenschaftsfond) via projects FWF I 199-N16, P21197-N16, as well as P22950-N16 and acknowledge also the RFBR project 09-02-91002 ANF_a. K. G. Kislyakova also acknowledges support by the RFBR project 08-02-00119 a, by the project NK-21P of the Education Ministry of the Russian Federation and by the EU grant \#228319 in the framework of the Project Europlanet RI-FP7. This work was also carried out under the support by the Interna- 
tional Space Science Institute (ISSI, Bern, Switzerland) and the ISSI team "Evolution of exoplanet atmospheres and their characterization". Finally the authors want to thank two anonymous referees for their comments and suggestions which helped to improve the manuscript.

\section{References}

Alibert, Y. B. C., W. Benz, G. Wuchterl, O. Grasset, C. Sotin, C. Eiroa, T. Henning, T. Herbst, L. Kaltenegger, A. Léger, R. Liseau, H. Lammer, C. Beichman, W. Danchi, M. Fridlund, J. Lunine, F. Paresce, A. Penny, A. Quirrenbach, H. Röttgering, F. Selsis, J. Schneider, D. Stam, G. Tinetti, and G. J. White, Origin and formation of planetary systems, Astrobiology, 10, 19-32, 2010.

Audard, M., M. Güdel, and E. F. Guinan, Implications from ExtremeUltraviolet observations for coronal heating of active stars, Astrophys. J. Lett., 513, L53-L56, 1999.

Audard, M., M. Güdel, J. J. Drake, and V. L. Kashyap, Extreme-Ultraviolet flare activity in late-type stars, Astrophys. J., 541, 396-409, 2000.

Ayres, T. R., Evolution of the solar ionizing flux, J. Geophys. Res., 102, 1641-1652, 1997.

Ayres, T. R., The cycles of $\alpha$ Centauri, Astrophys. J., 696, 1931-1949, 2009.

Bakalian, F., Production of hot nitrogen atoms in the martian thermosphere, Icarus, 183, 69-78, 2006.

Barnes, S. A., Ages for illustrative field stars using gyrochronology: Viability, limitations, and errors, Astrophys. J., 669, 1167-1189, 2007.

Bauer, S. J. and H. Lammer, Planetary Aeronomy, Springer Verlag, Heidelberg, New York, 2004

Bowman, B. R., W. K. Tobiska, F. A. Marcos, C. Y. Huang, C. S. Lin, and W. J. Burke, A new empirical thermospheric density model JB2008 using new solar and geomagnetic indices, AIAA/AAS Astrodynamics Specialist Conference, AIAA 2008-6438, pp. 19, 2008.

Catala, C. and the PLATO team, PLATO: PLAnetary Transits and Oscillations of stars, Exp. Astron., 23, 329-356, 2009a.

Catala, C. and the PLATO team, PLATO: PLAnetary Transits and Oscillations of stars, Commun. Asteroseismol., 158, 330-336, 2009b.

Chamberlain, J. W., Planetary coronae and atmospheric evaporation, Planet. Space. Sci., 11, 901-996, 1963.

Chassefière, E., Hydrodynamic escape of oxygen from primitive atmospheres: applications to the cases of Venus and Mars, Icarus, 124, 537$552,1996$.

Cnossen, I., J. Sanz-Forcada, F. Favata, O. Witasse, T. Zegers, and N. F. Arnold, Habitat of early life: Solar X-ray and UV radiation at Earth's surface 4-3.5 billion years ago, J. Geophys. Res., 112(E2), E02008, 2007.

Dehant, V., H. Lammer, Yu. N. Kulikov, J.-M. Grießmeier, D. Breuer, O. Verhoeven, Ö. Karatekin, T. van Hoolst, O. Korablev, and P. Lognonné, Planetary magnetic dynamo effect on atmospheric protection of early Earth and Mars, Space Sci. Rev., 129, 279-300, 2007.

DeWarf, L. E., K. M. Datin, and E. F. Guinan, X-ray, FUV, and UV observations of $\alpha$ Centauri B: Determination of long-term magnetic activity cycle and rotation period, Astron. Astrophys., 722, 343-357, 2010.

Dorren, J. D. and E. F. Guinan, HD 129333: The Sun in its infancy, Astrophys. J., 428, 805-818, 1994a.

Dorren, J. D. and E. F. Guinan, The Sun in time: Detecting and modelling magnetic inhomogenities on Solar-type stars, in The Sun as a Variable Star: Solar and Stellar Irradiance Variations, edited by Pap, J. M., C. Fröhlich, H. S. Hudson, and S. K. Solanki, 206-216 pp., Proceedings of IAU Colloquium 143, held in Boulder, Colorado, USA, June 2025, 1993, Cambridge University Press, Cambridge, U.K.; New York, U.S.A., 1994b.

Dorren, J. D., M. Guedel, and E. F. Guinan, X-Ray emission from the Sun in its youth and old age, Astrophys. J., 448, 431-436, 1995.

Eggen, O. J., Distribution and correlation of age, abundance, and motion of lower main sequence stars, Astron. J., 111, 466-475, 1996.

Eggenberger, P., C. Charbonnel, S. Talon, G. Meynet, A. Maeder, F. Carrier, and G. Bourban, Analysis of $\alpha$ Centauri AB including seismic constraints, Astron. Astrophys., 422, 235-246, 2004.

Eggenberger, P., A. Miglio, F. Carrier, J. Fernandes, and N. C. Santos, Analysis of 70 Ophiuchi AB including seismic constraints, Astron. Astrophys., 482, 631-638, 2008.

Ekenbäck, A., M. Holmström, P. Wurz, J.-M. Grießmeier, H. Lammer, F. Selsis, and T. Penz, Energetic neutral atoms around HD 209458b: Estimations of magnetospheric properties, Astrophys. J., 709, 670-679,
2010.

Favata, F., G. Micela, S. L. Baliunas, J. H. M. M. Schmitt, M. Güdel, F. R. Harnden, Jr., S. Sciortino, and R. A. Stern, High-amplitude, long-term X-ray variability in the solar-type star HD 81809: The beginning of an X-ray activity cycle?, Astron. Astrophys., 418, L13-L16, 2004.

Favata, F., G. Micela, S. Orlando, J. H. M. M. Schmitt, S. Sciortino, and J. Hall, The X-ray cycle in the solar-type star HD 81809. XMMNewton observations and implications for the coronal structure, Astron. Astrophys., 490, 1121-1126, 2008.

Fox, J. L. and A. B. Hać, Photochemical escape of oxygen from Mars: A comparison of the exobase approximation to a Monte Carlo method, Icarus, 204, 527-554, 2009.

Gaidos, E. J., M. Güdel, and G. A. Blake, The faint young Sun paradox: An observational test of an alternative solar model, Geophys. Res. Lett., 27, 501-503, 2000.

Gómez de Castro, A. I., I. Pagano, M. Sachkov, A. Lecavelier des Etangs, G. Piotto, R. González, and B. Shustov, Science with the world space observatory-ultraviolet, in New Quests in Stellar Astrophysics. II. Ultraviolet Properties of Evolved Stellar Populations, Proceedings of the International Conference held in Puerto Vallarta, Mexico, April 16-20, 2007, edited by Chavez, M., E. Bertone, D. Rosa-Gonzalez, and L. H. Rodriguez-Merino, 319-327 pp., Berlin, Heidelberg, Springer, 2009.

Grießmeier, J. M., U. Motschmann, A. Stadelmann, T. Penz, H. Lammer, F. Selsis, I. Ribas, E. F. Guinan, H. K. Biernat, and W. W. Weiss, The effect of tidal locking on the magnetospheric and atmospheric evolution of "Hot Jupiter", Astron. Astrophys., 425, 753-762, 2004.

Grießmeier, J.-M., S. Preusse, M. Khodachenko, and U. Motschmann, Exoplanetary radio emission under different stellar wind conditions, Planet. Space Sci., 55, 618-630, 2007.

Gröller, H., V. I. Shematovich, H. I. M. Lichtenegger, H. Lammer, M. Pfleger, Yu. N. Kulikov, W. Macher, U. V. Amerstorfer, and H. K. Biernat, Venus' atomic hot oxygen environment, J. Geophys. Res., 115, E12017, doi:10.1029/2010JE003697, 2010.

Güdel, M., Are coronae of magnetically active stars heated by flares?, Astrophys. J. Lett., 480, L121-L124, 1997.

Güdel, M., X-Ray Astronomy of stellar coronae, Astron. Astrophys. Rev., 12, 71-237, 2004.

Güdel, M., The Sun in Time: Activity and environment, Living Rev. Sol. Phys., 4(3), 2007.

Güdel, M., J. H. M. M. Schmitt, and A. O. Benz, Microwave emission from $\mathrm{X}$-ray bright solarlike stars: the F-G main sequence and beyond, Astron. Astrophys., 302, 775-787, 1995a.

Güdel, M., J. H. M. M. Schmitt, A. O. Benz, and N. M. Elias, II, The corona of the young solar analog EK Draconis, Astron. Astrophys., 301, 201-212, 1995b.

Güdel, M., E. F. Guinan, R. Mewe, J. S. Kaastra, and S. L. Skinner, A determination of the coronal emission measure distribution in the young solar analog EK Draconis from ASCA/EUVE spectra, Astrophys. J., 479, 416-426, 1997.

Güdel, M. and E. J. Gaidos, Deep radio observations of young solar analogs, in Cool Stars, Stellar Systems and the Sun: $11^{\text {th }}$ Cambridge Workshop, edited by Garćýa López, R. J., R. Rebolo, and M. R. Zapaterio Osorio, Proceedings of a meeting held at Puerto de la Cruz, Tenerife, Spain, 4-8 October 1999, 662-667 pp., ASP Conference Series 223, San Francisco, U.S.A., 2001

Güdel, M., M. Audard, V. L. Kashyap, J. J. Drake, and E. F. Guinan, Are coronae of magnetically active stars heated by flares? II. Extreme U1traviolet and X-ray flare statistics and the differential emission measure distribution, Astrophys. J., 582, 423-442, 2003.

Guinan, E. F. and I. Ribas, Our changing Sun: the role of solar nuclear evolution and magnetic activity on Earth's atmosphere and climate, in The Evolving Sun and its Influence on Planetary Environments, edited by Montesinos, B., A. Giménz, and E. F. Guinan, 85-107 pp, ASP 269, San Francisco, 2002.

Guinan, E. F., I. Ribas, and G. M. Harper, Far-Ultraviolet emissions of the Sun in Time: Probing solar magnetic activity and effects on evolution of paleoplanetary atmospheres, Astrophys. J., 594, 561-572, 2003.

Hall, L. A., J. E. Higgins, C. W. Chagnon, and H. E. Hinteregger, Solarcycle variation of the extreme ultraviolet radiation, J. Geophys. Res., 74, 4181-4183, 1969

Hedin, A. E., Extension of the MSIS thermosphere model into the middle and lower atmosphere, J. Geophys. Res., 96, 1159-1172, 1991.

Hodges, R. R., Jr., Distributions of hot oxygen for Venus and Mars, $J$. Geophys. Res., 105, 6971-6981, 2000.

Holmström, M., A. Ekenbäck, F. Selsis, T. Penz, H. Lammer, and P. Wurz, Energetic neutral atoms as the explanation for the high-velocity hydro- 
gen around HD 209458b, Nature, 451, 970-972, 2008.

Hunten, D. M., Atmospheric evolution of the terrestrial planets, Science, 259, 915-920, 1993.

Kasting, J. F., D. P. Whitmire, and R. T. Reynolds, Habitable zones around Main Sequence Stars, Icarus, 101, 108-128, 1993.

Khodachenko, M. L., I. Ribas, H. Lammer, J.-M. Grießmeier, M. Leitner, F. Selsis, C. Eiroa, A. Hanslmeier, H. K. Biernat, C. J. Farrugia, and H. O. Rucker, Coronal mass ejection (CME) activity of low mass $\mathrm{M}$ Stars as an important factor for the habitability of terrestrial exoplanets. I. CME impact on expected magnetospheres of Earth-like exoplanets in close-in habitable zones, Astrobiology, 7, 167-184, 2007.

Kim, J., A. F. Nagy, J. L. Fox, and T. E. Cravens, Solar cycle variability of hot oxygen atoms at Mars, J. Geophys. Res., 103, 29339-29342, 1998.

King, R. R., M. J. McCaughrean, D. Homeier, F. Allard, R.-D. Scholz, and $\mathrm{N}$. Lodieu, $\epsilon$ Indi Ba, Bb: A detailed study of the nearest known brown dwarfs, Astron. Astrophys., 510, A99, 2010.

Kjeldsen, H., T. R. Bedding, and J. Christensen-Dalsgaard, Measurements of stellar properties through Asteroseismology: A tool for planet transit studies, in Transiting Planets, Proceedings of the International Astronomical Union 2009, IAU Symposium, 253, 309-317 pp., Cambridge Univ. Press, Cambridge, UK, 2009.

Krestyanikova, M. A. and V. I. Shematovich, Stochastic models of hot planetary and satellite coronas: A hot oxygen corona of Mars, Sol. Syst. Res., 40, 384-392, 2006.

Kulikov, Y. N., H. Lammer, H. I. M. Lichtenegger, N. Terada, I. Ribas, C. Kolb, D. Langmayr, R. Lundin, E. F. Guinan, S. Barabash, and h. K. Biernat, Atmospheric and water loss from early Venus, Planet. Space Sci., 54, 1425-1444, 2006.

Kulikov, Y. N., H. Lammer, H. I. M. Lichtenegger, T. Penz, D. Breuer, T. Spohn, R. Lundin, and h. K. Biernat, A comparative study of the influence of the active young Sun on the early atmospheres of Earth, Venus and Mars, Space Sci.Rev., 129, 207-243, 2007.

Lammer, H. and S. J. Bauer, Nonthermal atmospheric escape from Mars and Titan, J. Geophys. Res., 96, 1819-1825, 1991.

Lammer, H., H. I. M. Lichtenegger, C. Kolb, I. Ribas, E. F. Guinan, R. Abart, and S. J. Bauer, Loss of water from Mars: Implications for the oxidation of the soil, Icarus, 106, 9-25, 2003.

Lammer, H., H. I. M. Lichtenegger, H. K. Biernat, N. V. Erkaev, I. L. Arshukova, C. Kolb, H. Gunell, A. Lukyanov, M. Holmström, S. Barabash, T. L. Zhang, and W. Baumjohann, Loss of hydrogen and oxygen from the upper atmosphere of Venus, Planet. Space Sci., 54, 1445-1456, 2006.

Lammer, H., H. I. M. Lichtenegger, Yu. N. Kulikov, J.-M. Grießmeier, N. Terada, N. V. Erkaev, H. K. Biernat, M. L. Khodachenko, I. Ribas, T. Penz, and F. Selsis, Coronal mass ejection (CME) activity of low mass $\mathrm{M}$ stars as an important factor for the habitability of terrestrial exoplanets. II. CME-induced ion pick up of Earth-like exoplanets in close-in habitable zones, Astrobiology, 7, 185-207, 2007.

Lammer, H., J. F. Kasting, E. Chassefière, R. E. Johnson, Yu. N. Kulikov, and F. Tian, Atmospheric escape and evolution of terrestrial planets and satellites, Space Sci. Rev., 139, 399-436, 2008.

Lammer, H., J. H. Bredehöft, A. Coustenis, M. L. Khodachenko, L. Kaltenegger, O. Grasset, D. Prieur, F. Raulin, P. Ehrenfreund, M. Yamauchi, J.-E. Wahlund, J.-M. Grießmeier, G. Stangl, C. S. Cockell, Yu. N. Kulikov, L. Grenfell, and H. Rauer, What makes a planet habitable?, Astron. Astrophys., 17, 181-249, 2009.

Lammer, H., A. Hanslmeier, J. Schneider, I. K. Stateva, M. Barthelemy, A. Belu, D. Bisikalo, M. Bonavita, V. Eybl, V. Coudé Du Foresto, M. Fridlund, R. Dvorak, S. Eggl, J.-M. Grießmeier, M. Güdel, E. Günther, W. Hausleitner, M. Holmström, E. Kallio, M. L. Khodachenko, A. A. Konovalenko, S. Krauss, L. V. Ksanfomality, Yu. N. Kulikov, K. Kyslyakova, M. Leitzinger, R. Liseau, E. Lohinger, P. Odert, E. Palle, A. Reiners, I. Ribas, H. O. Rucker, N. Sarda, J. Seckbach, V. I. Shematovich, A. Sozzetti, A. Tavrov, and M. Xiang-Grüß, Exoplanet status report: Observation, characterization and evolution of exoplanets and their host stars, Sol. Syst. Res., 44, 290-310, 2010.

Lammer, H., K. G. Kislyakova, M. Holmström, M. L. Khodachenko, and J.-M. Grießmeier, Hydrogen ENA-cloud observation and modeling as a tool to study star-exoplanet interaction, Astrophys. Space Sci., 335, 9-23, doi:10.1007/s10509-011-0604-2, 2011a.

Lammer, H., V. Eybl, K. G. Kislyakova, J. Weingrill, M. Holmström, M. L. Khodchenko, Yu. N. Kulikov, A. Reiners, M. Leitzinger, P. Odert, M. Xian Grüß, B. Dorner, M. Güdel, and A. Hanslmeier, UV transit observations of EUV-heated expanded thermospheres of Earth-like exoplanets around M-stars: Testing atmosphere evolution scenarios, Astrophys. Space Sci., 335, 39-50, doi:10.1007/s10509-011-0646-5, 2011b.
Lanza, A. F., I. Pagano, G. Leto, S. Messina, S. Aigrain, R. Alonso, M. Auvergne, A. Baglin, P. Barge, A. S. Bonomo, P. Boumier, A. Collier Cameron, M. Comparato, G. Cutispoto, J. R. de Medeiros, B. Foing, A. Kaiser, C. Moutou, P. S. Parihar, A. Silva-Valio, and W. W. Weiss, Magnetic activity in the photosphere of CoRoT-Exo-2a. Active longitudes and short-term spot cycle in a young Sun-like star, Astron. Astrophys., 493, 193-200, 2009.

Lecavelier des Etangs, A., D. Ehrenreich, A. Vidal-Madjar, G. E. Ballester, J.-M. Désert, R. Ferlet, G. Hébrard, D. K. Sing, K.-O. Tchakoumegni, and S. Udry, Evaporation of the planet HD 189733b observed in H I Lyman- $\alpha$, Astron. Astrophys., 514, A72, doi:10.1051/00046361/200913347, 2010.

Lichtenegger, H. I. M., H. Gröller, H. Lammer, Y. N. Kulikov, and V. I. Shematovich, On the elusive hot oxygen corona of Venus, Geophys. Res. Lett., 36, L10204, doi:10.1029/2009GL037575, 2009.

Lichtenegger, H. I. M., H. Lammer, J.-M. Grießmeier, Yu. N. Kulikov, P. von Paris, W. Hausleitner, S. Krauss, and H. Rauer, Aeronomical evidence for higher $\mathrm{CO}_{2}$ levels during Earth's Hadean epoch, Icarus, 210, 1-7, 2010 .

Lim, J. and S. M. White, Limits to mass outflows from late-type dwarf stars, Astrophys. J. Lett., 462, L91-L94, 1996.

Lundin, R., H. Lammer, and I. Ribas, Planetary magnetic fields and solar forcing: Implications for atmospheric evolution, Space Sci. Rev., 129, 245-278, 2007.

Mackay, D. H., M. Jardine, A. Collier Cameron, J. F. Donati, and G. A. J. Hussain, Polar caps on active stars: magnetic flux emergence and transport, Mon. Not. R. Astron. Soc., 354, 737-752, 2004.

Massi, M., J. Neidhöfer, G. Torricelli-Ciamponi, and F. Chiuderi-Drago, Activity cycles in UX ARIETIS, Astron. Astrophys., 332, 149-154, 1998.

Messina, S. and E. F. Guinan, Magnetic activity of six young solar analogues I. Star spot cycles from long-term photometry, Astron. Astrophys., 393, 225-237, 2002.

Messina, S. and E. F. Guinan, Magnetic activity of six young solar analogues II. Surface differential rotation from long-term photometry, Astron. Astrophys., 409, 1017-1030, 2003.

Montes, D., J. López-Santiago, M. C. Gálvez, M. J. Fernández-Figueroa, E. De Castro, and M. Cornide, Late-type members of young stellar kinematic groups-I. Single stars, Mon. Not. R. Astron. Soc., 328, 4563, 2001.

Nagy, A. F., T. E. Cravens, J. H. Yee, and A. I. F. Stewart, Hot oxygen atoms in the upper atmosphere of Venus, Geophys. Res. Lett., 8, 629$632,1981$.

Newkirk Jr., G., Solar variability on time scales of $10^{5}$ years to $10^{9.6}$ years, Geochim. Cosmochim. Acta Suppl., 13, 293-301, 1980.

Newman, M. J. and R. T. Rood, Implications of solar evolution for the Earth's early atmosphere, Science, 198, 1035-1037, 1977.

Öpik, E. J., Selective escape of gases, Geophys. J. R. Astron. Soc., 7, 490 $509,1963$.

Owen, T., The Martian atmosphere-Some unanswered questions, J. Mol. Evol., 14, 5-12, 1979.

Peres, G., S. Orlando, F. Reale, R. Rosner, and H. Hudson, The Sun as an X-ray star. II. Using the Yohkoh/Soft X-ray telescope-derived solar emission measure versus temperature to interpret stellar X-ray observations, Astrophys. J., 528, 537-551, 2000.

Pham, Lê Binh San, Ö. Karatekin, and V. Dehant, Effects of meteorite impacts on the atmospheric evolution of Mars, Astrobiology, 9, 45-54, 2009.

Phillips, R. J., M. T. Zuber, S. C. Solomon, M. P. Golombek, B. M Jakosky, W. B. Banerdt, D. E. Smith, R. M. E. Williams, B. M. Hynek, O. Aharonson, and S. A. Hauck, Ancient geodynamics and global-scale hydrology on Mars, Science, 291, 2587-2592, 2001.

Ribas, I., E. F. Guinan, M. Güdel, and M. Audard, Evolution of the solar activity over time and effects on planetary atmospheres. I. High-energy irradiances (1-1700 ̊̊), Astrophys. J., 622, 680-694, 2005.

Rieger, E., G. H. Share, D. J. Forrest, G. Kanbach, C. Reppin, and E. L. Chupp, Nature, 312, 623-625, 1984.

Roble, R. G., E. C. Ridley, and R. E. Dickinson, On the global mean structure of the thermosphere, J. Geophys. Res., 92, 8745-8758, 1987.

Robrade, J., J. H. M. M. Schmitt, and F. Favata, X-rays from $\alpha$-CentauriThe darkening of the solar twin, Astron. Astrophys, 442, 315-321, 2005.

Sagan, C. and G. Mullen, Earth and Mars: Evolution of atmospheres and surface temperatures, Science, 177, 52-56, 1972.

Sanz-Forcada, J., N. S. Brickhouse, and A. K. Dupree, The structure of stellar coronae in active binary systems, Astrophys. J. Suppl., 145, 147179, 2003. 
Sanz-Forcada, J., G. Micela, I. Ribas, A. M. T. Pollock, C. Eiroa, A. Velasco, E. Solano, and D. García-Álvarez, Evaporation in close planets produced by XUV radiation, Astron. Astrophys., 532, A6, 2011.

Scalo, J., L. Kaltenegger, A. G. Segura, M. Fridlund, I. Ribas, Yu. N. Kulikov, J. L. Grenfell, H. Rauer, P. Odert, M. Leitzinger, F. Selsis, M. L. Khodachenko, C. Eiroa, J. Kasting, and H. Lammer, M stars as targets for terrestrial exoplanet searches and biosignature detection, Astrobiology, 7, 85-166, 2007.

Schrijver, K. J. and A. M. Title, On the formation of polar spots in Sun-like stars, Astrophys. J., 551, 1099-1106, 2001.

Schüssler, M. and S. A. Solanki, Why rapid rotators have polar spots, Astron. Astrophys., 264, L13-L16, 1992.

Schüssler, M., P. Caligari, A. Ferriz-mas, S. A. Solanki, and M. Stix, Distribution of starspots on cool stars. I. Young and main sequence stars of $1 M_{\text {Sun }}$, Astron. Astrophys., 314, 503-512, 1996.

Sekiya, M., K. Nakazawa, and C. Hayashi, Dissipation of the primordial terrestrial atmosphere due to irradiation of the solar EUV, Prog. Theor. Phys., 64, 1968-1985, 1980a.

Sekiya, M., K. Nakazawa, and C. Hayashi, Dissipation of the rare gases contained in the primordial Earth's atmosphere, Earth Planet. Sci. Lett., 50, 197-201, 1980b.

Shematovich, V. I., D. Bisikalo, S. Krauss, W. Hausleitner, and H. Lammer, Influence of the hot oxygen corona on the satellite drag in the Earth's upper atmosphere, Sol. Syst. Res., 45, 238-246, 2011.

Shustov, B., M. Sachov, A. I. Gomez de Castro, I. Ana, and I. Pagano, WSO-UVultraviolet mission for the next decade, Astrophys. Space Sci., 320, 187-190, 2009.

Skumanich, A. and J. A. Eddy, Aspects of long-term variability in Sun and stars, in Solar Phenomena in Stars and Stellar Systems, 349-397 pp., D. Reidel Publishing Co., Dordrecht, 1981.

Smithtro, C. G. and J. J. Sojka, A new global average model of the coupled thermosphere and ionosphere, J. Geophys. Res., 110, A08305, doi:10.1029/2004JA010781, 2005a.

Smithtro, C. G. and J. J. Sojka, Behavior of the ionosphere and thermosphere subject to extreme solar cycle conditions, J. Geophys. Res., 110(A8), A08306, 2005b.

Soderblom, D. R., The ages of stars, Ann. Rev. Astron. Astrophys., 48, 581629, 2010.

Soderblom, D. R. and M. Mayor, Stellar kinematic groups. I. The Ursa Major group, Astron. J., 105, 226-249, 1993.

Stankov, A., M. Baldesarra, O. Piersanti, M. Fridlund, R. Lindberg, and N. Rando, Definition phase activities for ESA's Cosmic Vision mission PLATO, in Society of Photo-Optical Instrumentation Engineers (SPIE) Conference Series, edited by Oschmann, J. M., Jr., M. C. Clampin, and H. A. MacEwen, Proceedings of the SPIE, 7731, 77311M-77311M, pp. 2010.

Strassmeier, K. G., Starspots, Astron. Astrophys. Rev., 17, 251-308, 2009.

Sutton, E. K., J. M. Forbes, R. S. Nerem, and T. N. Woods, Neutral density response to the solar flares of October and November, 2003, Geophys. Res. Lett., 33, L22101, doi:10.1029/2006GL027737, 2006.

Telleschi, A., M. Güdel, K. Briggs, M. Audard, J.-U. Ness, and S. L. Skinner, Coronal evolution of the Sun in Time: High-resolution X-ray spectroscopy of solar analogs with different ages, Astrophys. J., 622, 653-679, 2005.

Terada, N., Yu. N. Kulikov, H. Lammer, H. I. M. Lichtenegger, T. Tanaka, H. Shinagawa, and T. Zhang, Atmosphere and water loss from early Mars under extreme solar wind and Extreme Ultraviolet conditions, Astrobiology, 9, 55-70, 2009.

Tian, F., Thermal escape from super Earth atmospheres in the habitable zones of M Stars, Astrophys. J., 703, 905-909, 2009.

Tian, F., O. B. Toon, A. A. Pavlov, and H. De Sterck, A hydrogen-rich early Earth atmosphere, Science, 308, 1014-1017, 2005.

Tian, F., J. F. Kasting, H. Liu, and R. G. Roble, Hydrodynamic planetary thermosphere model: 1 . The response of the Earth's thermosphere to extreme solar EUV conditions and the significance of adiabatic cooling, J. Geophys. Res., 113, doi:10.1029/2007JE002946, 2008a.

Tian, F., S. C. Solomon, L. Qian, J. Lei, and R. G. Roble, Hydrodynamic planetary thermosphere model: 2 . Coupling of an electron transport/energy deposition model, J. Geophys. Res., 113(E7), E07005, 2008 b.

Tian, F., J. F. Kasting, and S. C. Solomon, Thermal escape of carbon from the early Martian atmosphere, Geophys. Res. Lett., 36(2), L02205, 2009.

Tobiska, W. K., Recent solar extreme ultraviolet irradiance observations and modelling: A review, J. Geophys. Res., 98, 18879-18893, 1993.

Tobiska, W. K. and F. G. Eparvier, EUV97: Improvements to EUV irradiance modeling in the soft X-rays and FUV, Sol. Phys., 177, 147-159, 1998.

Valeille, A., M. R. Combi, V. Tenishev, S. W. Bougher, and A. F. Nagy, A study of suprathermal oxygen atoms in Mars upper thermosphere and exosphere over the range of limiting conditions, Icarus, 206, 18-27, 2010.

Vecchio, A., M. Laurenza, V. Carbone, and M. Storini, Quasi-biennial modulation of solar neutrino flux and solar and galactic cosmic rays by solar cyclic activity, Astrophys. J., 709, L1-L5, 2010.

Vidal-Madjar, A., A. Lecavelier des Etangs, J. M. Désert, G. E. Ballester, R. Ferlet, G. Hébrard, and M. Mayor, An extended upper atmosphere around the extrasolar planet HD209458b, Nature, 422, 143-146, 2003.

Walker, J. C. G., Evolution of the atmosphere of Venus, J. Atmos. Sci., 32, 1248-1256, 1975.

Walker, G. A. H., B. Croll, R. Kuschnig, A. Walker, S. M. Rucinski, J. M. Matthews, D. B. Guenther, A. F. J. Moffat, D. Sasselov, and W. W. Weiss, The differential rotation of $\kappa^{1}$ Ceti as observed by MOST, Astrophys. J., 659, 1611-1622, 2007.

Wargelin, B. J. and J. J. Drake, Observability of stellar winds from latetype dwarfs via charge exchange X-ray emission, Astrophys. J. Lett., 546, L57-L60, 2001.

Watson, A. J., T. M. Donahue, and J. C. G. Walker, The dynamics of a rapidly escaping atmosphere: applications to the evolution of Earth and Venus, Icarus, 48, 150-166, 1981.

Wood, B. E., Astrospheres and solar-like stellar winds, Living Rev. Sol. Phys., 1(2), 2004.

Wood, B. E. and J. L. Linsky, The Local ISM and its interaction with the winds of nearby late-type stars, Astrophys. J., 492, 788-803, 1998.

Wood, B. E., H.-R. Müller, G. Zank, and J. L. Linsky, Measured mass loss rates of solar-like stars as a function of age and activity, Astrophys. J., 574, 412-425, 2002.

Wood, B. E., H.-R. Müller, G. P. Zank, J. L. Linsky, and S. Redfield, New mass-loss measurements from astrospheric Ly- $\alpha$ absorption, Astrophys. $J ., 628$, L143-L146, 2005.

Zahnle, K. J. and J. C. G. Walker, The evolution of solar ultraviolet luminosity, Rev. Geophys., 20, 280-292, 1982.

Zaqarashvili, T. V., M. Carbonell, R. Oliver, and J. L. Ballester, Magnetic Rossby waves in the solar tachocline and Rieger-type periodicities, Astrophys. J., 709, 749-758, 2010a.

Zaqarashvili, T. V., M. Carbonell, R. Oliver, and J. L. Ballester, Quasibiennial oscillations in the solar tachocline caused by magnetic Rossby wave instabilities, Astrophys. J. Lett., 724, L95-L98, 2010 b.

Zaqarashvili, T. V., R. Oliver, J. L. Ballester, M. Carbonell, M. L. Khodachenko, H. Lammer, M. Leitzinger, and P. Odert, Rossby waves and polar spots in rapidly rotating stars: implications for stellar wind evolution, Astron. Astrophys., 532, A139, 2011.

H. Lammer (e-mail: helmut.lammer@oeaw.ac.at), M. Güdel, Y. Kulikov, I. Ribas, T. V. Zaqarashvili, M. L. Khodachenko, K. G. Kislyakova, H. Gröller, P. Odert, M. Leitzinger, B. Fichtinger, S. Krauss, W. Hausleitner, M. Holmström, J. Sanz-Forcada, H. I. M. Lichtenegger, A. Hanslmeier, V. I. Shematovich, D. Bisikalo, H. Rauer, and M. Fridlund 